\title{
HÖLDER REGULARITY OF QUASIMINIMIZERS UNDER GENERALIZED GROWTH CONDITIONS
}

\author{
PETTERI HARJULEHTO, PETER HÄSTÖ AND OLLI TOIVANEN
}

\begin{abstract}
We prove Harnack's inequality for local (quasi)minimizers in generalized Orlicz spaces without polynomial growth or coercivity conditions. As a consequence, we obtain the local Hölder continuity of local (quasi)minimizers. The results include as special cases standard, variable exponent and double phase growth.
\end{abstract}

\section{INTRODUCTION}

In the calculus of variations one studies existence and properties of solutions to minimization problems such as

$$
\min _{u \in W^{1,1}} \int F(x,|\nabla u|) d x
$$

Classical techniques, by De Giorgi and Moser, cover both the linear case and the $p$-growth case, where $F(t) \approx t^{p}$. Marcellini [31,32] developed the theory of $(p, q)$-growth, which is based on the growth assumption $t^{p}-1 \lesssim F(x, t) \lesssim t^{q}+1, q>p$. In this case, results hold provided the ratio $\frac{q}{p}$ is sufficiently close to 1 . For instance, the minimizer is Lipschitz continuous if $\frac{q}{p} \leqslant 1+\frac{2}{n-2}, n>2$ being the dimension. Zhikov [43, 44] studied such minimizers as models of anisotropic materials and also observed that they exhibit the socalled Lavrentiev phenomenon whereby minimizers do not have improved regularity and may even be discontinuous. Marcellini's theory provides maximal local flexibility (since there is a gap between the upper and lower bounds), but it is rigid globally, since the lower bound at one location restricts the upper bound everywhere else.

The opposite is true for variable exponent growth, i.e. $F(x, t) \approx t^{p(x)}$. This theory provides results also in the case when $\frac{\sup p}{\inf p}>1+\frac{2}{n-2}$ but conversely requires some continuity properties of $p$, cf. [33]. Such problems have been vigorously studied in recent years [10,12].

In the variable exponent case, the change in the anisotropy (growth rate) is gradual owing to the continuity of $p$. For instance, in electrorheological fluid dynamics, where the anisotropy depends on the smooth electrical field, this is a reasonable assumption [40]. In other situations, such as composite materials, a more clear-cut transition is better. To this end, Baroni, Colombo and Mingione [3, 4, 7, 8, 9] have studied the double phase functional

$$
F(x, t)=t^{p}+a(x) t^{q}, \quad q>p,
$$

which has the property that the growth rate changes abruptly from $p$ to $q$ in the sets $\{a=0\}$ and $\{a>0\}$. They have nevertheless managed to build of regularity theory for minimizers under the assumption that $a \in C^{\alpha}$ where either $\frac{q}{p} \leqslant 1+\frac{\alpha}{n}$ or $\frac{q}{p} \leqslant 1+\frac{\alpha}{p}$ is required.

Date: October 3, 2016.

2010 Mathematics Subject Classification. 35J60, 35B65, 49J40, 46 E35.

Key words and phrases. Harnack's inequality, Dirichlet energy integral, minimizer, local minimizer, generalized Orlicz space, Musielak-Orlicz spaces, continuity of solution, nonstandard growth, variable exponent, double phase. 
The reason that variable exponent research thrived while little harmonic analysis was done in generalized Orlicz spaces was the belief that many classical results can be obtained in the former setting but not the latter. However, this belief has been challenged recently, based on new techniques that were developed and perfected in the context of variable exponent spaces $[11,13,19,20,24,27,28,29,30,35,36,42]$.

We have developed a set of assumptions, imaginatively called (A0), (A1) and (A2), which appear to very neatly capture all previously mentioned results. (A2) pertains to unbounded domains and will not be used in this paper; (A0) is just a restriction to the un-weighted case and is likely to be of a technical nature. The central condition (A1) corresponds to local logHölder continuity in the variable exponent case (simple calculation) and to the restriction $\frac{q}{p} \leqslant 1+\frac{\alpha}{n}$ in the double phase case (see Theorem 4.7 in [24]). Both are known to be the optimal continuity moduli in their respective settings.

In this paper, we introduce a new condition, called (A1- $n$ ). In the variable exponent setting it too corresponds to local log-Hölder continuity and for the double phase functional it says that $\frac{q}{p} \leqslant 1+\frac{\alpha}{p}$, as in Baroni-Colombo-Mingione. The fact that our assumptions agree with known optimal assumptions in two very disparate cases strongly suggests that they form a reasonable basis for a general theory. For the precise definition of the assumptions, see Section 2.1.

Here we take the first step towards a regularity theory in generalized Orlicz spaces by proving Harnack's inequality and Hölder continuity of local quasiminimizers, defined as follows (see Section 2 for further definitions). Note that any minimizer of (1.1) with

$$
F(x, t) \approx \varphi(x, t)
$$

is a local quasiminimizer and hence covered by our results.

Definition 1.2. Let $\varphi \in \Phi_{w}(\Omega)$. A function $u \in W_{\mathrm{loc}}^{1, \varphi(\cdot)}(\Omega)$ is a local quasiminimizer of the $\varphi(\cdot)$-energy in $\Omega$ if there exists a constant $K \geqslant 1$ such that

$$
\int_{\{v \neq 0\}} \varphi(x,|\nabla u|) d x \leqslant K \int_{\{v \neq 0\}} \varphi(x,|\nabla(u+v)|) d x
$$

for all $v \in W^{1, \varphi(\cdot)}(\Omega)$ with spt $v:=\overline{\{v \neq 0\}} \subset \Omega$.

Using Theorem 4.11 for $u$ and $-u$, we obtain the following theorem.

Theorem 1.3 (Local boundedness). Assume that $\varphi \in \Phi_{w}(\Omega)$ satisfies (A0), (A1), (aInc) and $($ aDec). Then every local quasiminimizer of the $\varphi(\cdot)$-energy is locally bounded.

Our main result, which we obtain by combining Corollaries 5.9 and 6.5, is:

Theorem 1.4 (Harnack's inequality). Assume that $\varphi \in \Phi_{w}(\Omega)$ satisfies (AO), (A1), (Al-n), (aDec) and (aInc). Suppose that $u \in W_{\mathrm{loc}}^{1, \varphi(\cdot)}(\Omega)$ is a non-negative local quasiminimizer of the $\varphi(\cdot)$-energy. For compact $K \Subset \Omega$, there exists $R_{0}$ (cf. Theorem 5.7) such that

$$
\underset{x \in Q_{R}}{\operatorname{ess} \sup } u(x) \leqslant C\left(\underset{x \in Q_{R}}{\operatorname{essinf}} u(x)+R\right)
$$

for all $R \in\left(0, R_{0}\right]$ with and cubes $Q_{6 R} \Subset \Omega$ centered in $K$. The constant $C$ depends only on the parameters from the assumptions, the dimension $n$, and the norm $\|u\|_{L^{\infty}\left(Q_{2 R}\right)}$.

If $u \in W^{1, \varphi(\cdot)}(\Omega)$ in the previous theorem, then $R_{0}$ can be chosen for all of $\Omega$, not just a compact subset.

By standard arguments, the previous Harnack inequality implies the Hölder continuity of the function. 
Corollary 1.5 (Hölder continuity). Assume that $\varphi \in \Phi_{w}(\Omega)$ satisfies (AO), (Al), (Al-n), $(\mathrm{aDec})$ and (aInc). If $u$ is a local quasiminimizer of the $\varphi(\cdot)$-energy, then $u \in C_{\mathrm{loc}}^{\alpha}(\Omega)$ for some $\alpha>0$.

In addition to being a natural generalization which covers results from both variable exponent and Orlicz spaces, the study of nonstandard growth problems can be motivated by applications to image processing [1, 6, 21], fluid dynamics [40, 41] and differential equations $[2,14,17,18,37,38]$

The structure of this paper is as follows. First we recall some basic properties of generalized Orlicz spaces in Section 2. Then we introduce the assumptions (Ax) and prove several auxiliary results related to them (Section 2.1). In Section 3 we prove Sobolev and maximal inequalities, both in modular form. Since modular inequalities are known not to be generally true in this context, both have been crafted to work in the specific situation arising in this paper.

The proof of the Harnack inequality uses De Giorgi's method. In Section 4, we derive a Caccioppoli inequality and prove the local boundedness of solutions by iteration. This is the main part of the proof. While the general scheme is familiar, several technical innovations are necessary to apply it in this context. Furthermore, the resultant upper bound is quantitatively poor: the right-hand side is not homogeneous and the constant of comparison blows up when the radius tends to zero. These short-comings are remedied in Section 5 via a scaling trick from the Orlicz setting and bootstrapping the boundedness result of the preceding section. It is at this junction that we need the new assumption (A1- $n$ ). It is interesting to note that Mingione et al. use the corresponding double phase assumption precisely when dealing bounded solutions. The proof is concluded in Section 6 by establishing the weak Harnack inequality.

\section{PRoperties of GENERALIZED $\Phi$-FUnCTIONS}

Generalized Orlicz spaces $L^{\varphi(\cdot)}$ have been studied since the 1940s. A major synthesis of functional analysis in these spaces is given in the 1983 monograph of Musielak [34], hence the alternative name Musielak-Orlicz spaces. In this section we present pertinent aspects of the theory of generalized Orlicz spaces from a modern point-of-view which emphasizes properties that are invariant under equivalence of $\Phi$-functions. This approach is inspired by our Japanese colleagues [27], although our assumptions are more general than theirs, cf. [24].

By $\Omega \subset \mathbb{R}^{n}$ we denote a bounded domain, i.e. an open and connected set. The notation $f \lesssim g$ means that there exists a constant $C>0$ such that $f \leqslant C g$. The notation $f \approx g$ means that $f \lesssim g \lesssim f$. By $c$ we denote a generic constant whose value may change between appearances. A function $f$ is almost increasing if there exists a constant $L \geqslant 1$ such that $f(s) \leqslant L f(t)$ for all $s \leqslant t$ (abbreviated $L$-almost increasing). Almost decreasing is defined similarly.

Definition 2.1. We say that $\varphi: \Omega \times[0, \infty) \rightarrow[0, \infty]$ is a weak $\Phi$-function, and write $\varphi \in \Phi_{w}(\Omega)$, if

- For every $t \in[0, \infty)$ the function $x \mapsto \varphi(x, t)$ is measurable and for every $x \in \Omega$ the function $t \mapsto \varphi(x, t)$ is increasing.

- $\varphi(x, 0)=\lim _{t \rightarrow 0^{+}} \varphi(x, t)=0$ and $\lim _{t \rightarrow \infty} \varphi(x, t)=\infty$ for every $x \in \Omega$.

- The function $t \mapsto \frac{\varphi(x, t)}{t}$ is almost increasing for $t>0$ uniformly in $\Omega$. "Uniformly" means that the constant is independent of $x$. 
Two functions $\varphi$ and $\psi$ are equivalent, $\varphi \simeq \psi$, if there exists $L \geqslant 1$ such that $\psi\left(x, \frac{t}{L}\right) \leqslant$ $\varphi(x, t) \leqslant \psi(x, L t)$ for every $x \in \Omega$ and every $t>0$. Equivalent $\Phi$-functions give rise to the same space with comparable norms.

We say that $\varphi$ is doubling if there exists a constant $L \geqslant 1$ such that $\varphi(x, 2 t) \leqslant L \varphi(x, t)$ for every $x \in \Omega$ and every $t>0$. If $\varphi$ is doubling with constant $L$, then by iteration

$$
\varphi(x, t) \leqslant L^{2}\left(\frac{t}{s}\right)^{Q} \varphi(x, s)
$$

for every $x \in \Omega$ and every $0<s<t$, where $Q=\log _{2}(L)$. For the proof see for example [5, Lemma 3.3 , p. 66]. If $\varphi$ is doubling, then (2.2) yields that $\simeq$ implies $\approx$. On the other hand, $\approx$ always implies $\simeq$ since the function $t \mapsto \frac{\varphi(x, t)}{t}$ is almost increasing; hence $\simeq$ and $\approx$ are equivalent in the doubling case. Note that doubling also yields that $\varphi(x, t+s) \leqslant$ $L \varphi(x, t)+L \varphi(x, s)$.

Doubling functions have many nice properties, including the following. Note that the claims in the lemma are to be understood with respect of the second variable of $\varphi$. Similarly, by $\varphi^{-1}$ we denote the left-continuous inverse of a weak $\Phi$-function $\varphi$,

$$
\varphi^{-1}(\tau):=\inf \{t \geqslant 0: \varphi(t) \geqslant \tau\} .
$$

Lemma 2.3. If $\varphi \in \Phi_{w}(\Omega)$ is doubling, then there exists $\psi \in \Phi_{w}(\Omega)$ with $\psi \approx \varphi$ which is strictly increasing and continuous, hence a bijection.

Proof. By [20, Proposition 2.3], there exists $\psi \in \Phi_{w}(\Omega)$ with $\psi \simeq \varphi$ which is convex. Then $\psi$ is also doubling and $\psi \approx \varphi$. It follows from doubling that $\psi(x, t) \in(0, \infty)$ for every $t>0$. A finite convex function is continuous. Convexity implies that $\frac{\psi(x, t)}{t}$ is increasing which combined with $\psi(x, t)>0$ implies strict increasing.

The conjugate $\Phi$-function $\varphi^{*}$ is defined by the formula

$$
\varphi^{*}(x, t):=\sup _{s \geqslant 0}(s t-\varphi(x, s)) .
$$

Young's inequality follows directly from this definition:

$$
s t \leqslant \varphi(x, s)+\varphi^{*}(x, t) .
$$

2.1. Assumptions. Let us write $\varphi_{B}^{+}(t):=\sup _{x \in B} \varphi(x, t)$ and $\varphi_{B}^{-}(t):=\inf _{x \in B} \varphi(x, t)$; and abbreviate $\varphi^{ \pm}:=\varphi_{\Omega}^{ \pm}$.

Assume following [24] that there exist $\beta, \sigma>0$ such that $\varphi^{+}(\beta) \leqslant 1 \leqslant \varphi^{-}(\sigma)$ and

$$
\varphi_{B}^{+}(\beta t) \leqslant \varphi_{B}^{-}(t) \quad \text { when } \quad t \in\left[\sigma,\left(\varphi_{B}^{-}\right)^{-1}\left(\frac{1}{|B|}\right)\right]
$$

for every ball $B \subset \Omega$. Then the rescaling $\widetilde{\varphi}(x, t):=\varphi\left(x, \frac{t}{\sigma}\right)$ satisfies the same conditions with $\sigma=1$. Thus we arrive at the following assumptions.

(A0) There exists $\beta \in(0,1)$ such that $\varphi^{+}(\beta) \leqslant 1 \leqslant \varphi^{-}(1)$.

(A1) There exists $\beta \in(0,1)$ such that, for every ball $B \subset \Omega$,

$$
\varphi_{B}^{+}(\beta t) \leqslant \varphi_{B}^{-}(t) \quad \text { when } \quad t \in\left[1,\left(\varphi_{B}^{-}\right)^{-1}\left(\frac{1}{|B|}\right)\right] .
$$

(A1-n) There exists $\beta \in(0,1)$ such that, for every ball $B \subset \Omega$,

$$
\varphi_{B}^{+}(\beta t) \leqslant \varphi_{B}^{-}(t) \quad \text { when } t \in\left[1, \frac{1}{\operatorname{diam} B}\right] .
$$

(aInc) There exist $\gamma^{-}>1$ and $L \geqslant 1$ such that $t \mapsto \frac{\varphi(x, t)}{t^{-}}$is $L$-almost increasing in $(0, \infty)$. (aDec) There exist $\gamma^{+}>1$ and $L \geqslant 1$ such that $t \mapsto \frac{\varphi(x, t)}{t^{\gamma^{+}}}$is $L$-almost decreasing in $(0, \infty)$. 
We write (Inc) if the ratio is increasing rather than just almost increasing, similarly for (Dec). Note that $\gamma^{+} \geqslant \gamma^{-}$. All these assumptions are invariant under equivalence of $\Phi$-functions, modulo the scaling $(\sigma=1)$ mentioned in the beginning of the section. Note that the optimal $\gamma^{-}$and $\gamma^{+}$correspond to the lower and upper Matuszewska-Orlicz indexes, respectively.

Example 2.5. Let us consider the assumptions in some important special cases, namely variable exponent growth and double phase growth. The next table contains a interpretation of the assumptions for four $\Phi$-functions. Note that in many cases the condition in the special case is just an nearly optimal sufficient condition: for instance, in the variable exponent case $p \in C^{\log }$ implies (A1), and no worse continuity modulus is sufficient, but there may be exponents $p \notin C^{\log }$ for which (A1) nevertheless holds [4, 7, 24, 39].

\begin{tabular}{l|ccccc}
$\varphi(x, t)$ & $(\mathrm{A} 0)$ & $(\mathrm{A} 1)$ & $(\mathrm{A} 1-n)$ & $(\mathrm{aInc})$ & $(\mathrm{aDec})$ \\
\hline$t^{p(x)} a(x)$ & $a \approx 1$ & $p \in C^{\log }$ & $p \in C^{\log }$ & $p^{-}>1$ & $p^{+}<\infty$ \\
$t^{p(x)} \log (e+t)$ & true & $p \in C^{\log }$ & $p \in C^{\log }$ & $p^{-}>1$ & $p^{+}<\infty$ \\
$t^{p}+a(x) t^{q}$ & $a \in L^{\infty}$ & $a \in C^{\frac{n}{p}(q-p)}$ & $a \in C^{q-p}$ & $p>1$ & $q<\infty$ \\
$t^{p}+a(x) t^{p} \log (e+t)$ & $a \in L^{\infty}$ & $a \in C^{\log }$ & $a \in C^{\log }$ & $p>1$ & $p<\infty$
\end{tabular}

The (almost) decreasing condition is equivalent to doubling. (Similarly, (aInc) is equivalent to the $\nabla_{2}$ condition, which is not used in this paper.)

Lemma 2.6. Let $\varphi \in \Phi_{w}$.

(1) Doubling is equivalent to (aDec).

(2) If $\varphi$ is convex, then doubling is equivalent to (Dec).

Proof. By the (aDec) or (Dec) condition,

$$
\frac{\varphi(2 t)}{(2 t)^{\gamma^{+}}} \lesssim \frac{\varphi(t)}{t^{\gamma^{+}}}
$$

so $\varphi(2 t) \lesssim 2^{\gamma^{+}} \varphi(t)$, i.e. $\varphi$ is doubling.

If $\varphi$ is doubling, then by (2.2), (aDec) holds with with $Q=\log _{2}(L)$, where $L$ is the doubling constant. Hence (1) is proved.

Assume that $\varphi(t)$ is convex and doubling. If $t \geqslant 2 s$, then $L^{2}\left(\frac{t}{s}\right)^{Q} \leqslant\left(\frac{t}{s}\right)^{Q^{\prime}}$, where $Q^{\prime}=$ $\max \left\{Q, \frac{2 \ln L}{\ln 2}\right\}$, and (Dec) follows from (2.2). Suppose that $t<s<2 t$ and $s^{-\gamma} \varphi(s)>$ $t^{-\gamma} \varphi(t)$ for some $\gamma>1$. It follows by doubling and convexity for the first and second inequalities, respectively, that

$$
L \geqslant \frac{\varphi(2 t)}{\varphi(t)} \geqslant \frac{\varphi(s)-\varphi(t)}{s-t} \frac{t}{\varphi(t)} \geqslant \frac{(s / t)^{\gamma}-1}{s / t-1} \geqslant \gamma
$$

Thus $s^{-\gamma} \varphi(s) \leqslant t^{-\gamma} \varphi(t)$ when $\gamma>L$, and it follows that (Dec) holds with $\gamma>\max \left\{L, Q^{\prime}\right\}$.

Lemma 2.7 (Lemma 3.3, [19]). Assumption (A0) implies that $\varphi^{-1}(x, 1) \approx 1$.

Furthermore, (A0) and (aDec) imply that $\varphi(x, 1) \lesssim \beta^{-\gamma^{+}} \varphi(x, \beta) \leqslant \beta^{-\gamma^{+}}$, so

$$
\varphi(x, 1) \approx 1 \text {. }
$$

Lemma 2.8. Suppose that $\varphi \in \Phi_{w}(\Omega)$ and $\varphi(x, t)$ is a bijection in tor $\varphi$ is doubling.

- (aDec) holds if and only if $\varphi^{-1}(x, t) / t^{1 / \gamma^{+}}$is almost increasing uniformly in $\Omega$.

- (aInc) holds if and only if $\varphi^{-1}(x, t) / t^{1 / \gamma^{-}}$is almost decreasing uniformly in $\Omega$. 
Proof. Assume first that $\varphi$ is a bijection. Let $t=\varphi^{-1}(x, s)$ and $p \geqslant 1$. Then

$$
\frac{\varphi(x, t)}{t^{p}}=\frac{s}{\varphi^{-1}(x, s)^{p}}=\left(\frac{\varphi^{-1}(x, s)}{s^{\frac{1}{p}}}\right)^{-p} .
$$

Since $\varphi^{-1}$ is increasing, $t$ is an increasing function of $s$. Thus the left-hand side is increasing in $t$ if and only if the right-hand side is increasing in $s$. Taking into account the negative exponent on the right-hand side, this gives the result.

Assume then that $\varphi$ is doubling. By Lemma 2.3 we obtain $\psi \approx \varphi$ that is a bijection. The first part of the proof give the claims for $\psi$ but this yields the claims for $\varphi$.

$(\mathrm{A} 1-n)$ follows from (A0), (A1) and (aDec) if $\gamma^{+} \leqslant n$ (hence the $n$ in the notation):

Lemma 2.9. Assume that $\varphi \in \Phi_{w}(\Omega)$ satisfies (AO), (Al) and (aDec). If $\gamma^{+} \leqslant n$, then $\varphi$ satisfies (Al-n).

Proof. (A1-n) follows from (A1) if $\left(\varphi_{B}^{-}\right)^{-1}\left(\frac{1}{|B|}\right) \geqslant \frac{1}{\operatorname{diam} B}$. For every $x \in B$ we have $\left(\varphi_{B}^{-}\right)^{-1}\left(\frac{1}{|B|}\right) \geqslant \varphi^{-1}\left(x, \frac{1}{|B|}\right)$. Since $\varphi$ is almost decreasing with exponent $\gamma^{+}$, Lemma 2.8 implies that $\varphi^{-1}(x, t) / t^{1 / \gamma^{+}}$is almost increasing. This and Lemma 2.7 give that

$$
\frac{\varphi^{-1}\left(x, \frac{1}{|B|}\right)}{|B|^{-1 / \gamma^{+}}} \gtrsim \frac{\varphi^{-1}(x, 1)}{1^{1 / \gamma^{+}}} \approx 1
$$

and hence

$$
\varphi^{-1}\left(x, \frac{1}{|B|}\right) \gtrsim \frac{1}{|B|^{1 / \gamma^{+}}} \approx \frac{1}{\operatorname{diam}(B)^{n / \gamma^{+}}} \gtrsim \frac{1}{\operatorname{diam} B}
$$

since $|B| \leqslant 1$ and $\frac{n}{\gamma^{+}} \geqslant 1$. Thus there exists $\alpha \in(0,1]$ such that

$$
\varphi_{B}^{+}(\beta t) \leqslant \varphi_{B}^{-}(t) \quad \text { when } \quad t \in\left[1, \frac{\alpha}{\operatorname{diam} B}\right] .
$$

When $t \in\left(\frac{\alpha}{\operatorname{diam} B}, \frac{1}{\operatorname{diam} B}\right]$ we obtain by the previous case that

$$
\varphi_{B}^{+}(\alpha \beta t) \leqslant \varphi_{B}^{-}(\alpha t) \leqslant \varphi_{B}^{-}(t) .
$$

Hence (A1-n) holds with a constant $\alpha \beta$.

If $Q \subset \mathbb{R}^{n}$ is a ball of cube, then we denote by $c Q$ a ball or cube with the same center and $c$-fold diameter.

Lemma 2.10. Let $\varphi \in \Phi_{w}(\Omega)$ satisfy $(A 1)$ or $(A 1-n)$. There exists $\beta \in(0,1)$ such that, for every cube $\sqrt{n} Q \subset \Omega$,

$$
\varphi_{Q}^{+}(\beta t) \leqslant \varphi_{Q}^{-}(t) \quad \text { when } t \in[1, M]
$$

where $M:=\left(\varphi_{Q}^{-}\right)^{-1}\left(\frac{1}{|\sqrt{n} Q|}\right)$ for $(A 1)$ and $M:=\frac{1}{\operatorname{diam} Q}$ for $(A 1-n)$.

Proof. Let $B$ be the smallest ball containing $Q$. Since $B \subset \sqrt{n} Q,|B| \leqslant|\sqrt{n} Q|$. Assumption (A1) yields that

$$
\varphi_{Q}^{+}(\beta t) \leqslant \varphi_{B}^{+}(\beta t) \leqslant \varphi_{B}^{-}(t) \leqslant \varphi_{Q}^{-}(t) \quad \text { when } \quad t \in\left[1,\left(\varphi_{B}^{-}\right)^{-1}\left(\frac{1}{|B|}\right)\right] .
$$

The first claim follows for this since $\left(\varphi_{Q}^{-}\right)^{-1}\left(\frac{1}{|\sqrt{n} Q|}\right) \leqslant\left(\varphi_{B}^{-}\right)^{-1}\left(\frac{1}{|B|}\right)$. The second claim follows analogously from (A1- $n)$ since $\operatorname{diam} B=\operatorname{diam} Q$.

Lemma 2.11. If $\varphi \in \Phi_{w}(\Omega)$ satisfies (Al) and is a bijection, then there exists $\beta \in(0,1)$ such that $\beta \varphi^{-1}(x, t) \leqslant \varphi^{-1}(y, t)$ for every $t \in\left[1, \frac{1}{|\sqrt{n} Q|}\right]$, every $x, y \in Q$ and every cube $Q$ with $\sqrt{n} Q \subset \Omega$ and $|\sqrt{n} Q| \leqslant 1$. 
Proof. Let $t \in\left[1, \frac{1}{|\sqrt{n} Q|}\right]$. By Lemma 2.7, $\varphi^{-1}(y, t) \in\left[\alpha, \varphi^{-1}\left(y, \frac{1}{|\sqrt{n} Q|}\right)\right]$ for some $\alpha \in(0,1]$. If $\varphi^{-1}(y, t) \in\left[1, \varphi^{-1}\left(y, \frac{1}{|\sqrt{n} Q|}\right)\right]$ then by Lemma 2.10

$$
\varphi\left(x, \beta \varphi^{-1}(y, t)\right) \leqslant \varphi\left(y, \varphi^{-1}(y, t)\right)=t
$$

and the claim follows from this by taking $\varphi^{-1}(x, \cdot)$ of both sides.

Assume then that $\varphi^{-1}(y, t) \in[\alpha, 1)$, Since $\varphi$ is bijection, there exists $\xi$ such that $\varphi(x, \xi)=$ 1. We obtain

$$
\varphi\left(x, \min \{1, \xi\} \varphi^{-1}(y, t)\right) \leqslant \varphi(x, \xi)=1 \leqslant t
$$

and the claim follows from this by taking $\varphi^{-1}(x, \cdot)$ of both sides. The claim follows by choosing the constant to be $\min \{\beta, \xi\}$.

\section{FUnCTION SPACES AND INEQUALITIES}

The generalized Orlicz and Orlicz-Sobolev spaces have been studied with our assumptions in [20]. We recall some definitions. We denote by $L^{0}(\Omega)$ the set of measurable functions in $\Omega$.

Definition 3.1. Let $\varphi \in \Phi_{w}(\Omega)$ and define the modular $\varrho_{\varphi(\cdot)}$ for $f \in L^{0}(\Omega)$ by

$$
\varrho_{\varphi(\cdot)}(f):=\int_{\Omega} \varphi(x,|f(x)|) d x .
$$

The generalized Orlicz space, also called Musielak-Orlicz space, is defined as the set

$$
L^{\varphi(\cdot)}(\Omega):=\left\{f \in L^{0}(\Omega): \lim _{\lambda \rightarrow 0^{+}} \varrho_{\varphi(\cdot)}(\lambda f)=0\right\}
$$

equipped with the (Luxemburg) norm

$$
\|f\|_{L^{\varphi(\cdot)}(\Omega)}:=\inf \left\{\lambda>0: \varrho_{\varphi(\cdot)}\left(\frac{f}{\lambda}\right) \leqslant 1\right\} .
$$

If the set is clear from the context we abbreviate $\|f\|_{L^{\varphi(\cdot)}(\Omega)}$ by $\|f\|_{\varphi(\cdot) \text {. }}$.

Hölder's inequality holds in generalized Orlicz spaces with a constant 2, without restrictions on the $\Phi$-function [12, Lemma 2.6.5]:

$$
\int_{\Omega}|f||g| d x \leqslant 2\|f\|_{\varphi(\cdot)}\|g\|_{\varphi^{*}(\cdot) \cdot}
$$

Definition 3.2. A function $u \in L^{\varphi(\cdot)}(\Omega)$ belongs to the Orlicz-Sobolev space $W^{1, \varphi(\cdot)}(\Omega)$ if its weak partial derivatives $\partial_{1} u, \ldots, \partial_{n} u$ exist and belong to $L^{\varphi(\cdot)}(\Omega)$.

Definition 3.3. $W_{0}^{1, \varphi(\cdot)}(\Omega)$ is the closure of $C_{0}^{\infty}(\Omega)$ in $W^{1, \varphi(\cdot)}(\Omega)$.

If $\varphi \in \Phi_{w}$ satisfies (A0) and (aInc), and $\Omega \subset \mathbb{R}^{n}$ is bounded, then we have the inclusions $L^{\varphi(\cdot)}(\Omega) \hookrightarrow L^{\gamma^{-}}(\Omega), W^{1, \varphi(\cdot)}(\Omega) \hookrightarrow W^{1, \gamma^{-}}(\Omega)$ and $W_{0}^{1, \varphi(\cdot)}(\Omega) \hookrightarrow W_{0}^{1, \gamma^{-}}(\Omega)$. For the proofs see Lemmas 4.4, 6.2 and 6.9 in [20].

We need the following fact regarding Sobolev functions. The problem is that smooth functions are not necessary dense in the Orlicz-Sobolev space and in this case our definition for zero boundary values Orlicz-Sobolev space is deficient.

Lemma 3.4. Let $\Omega \subset \mathbb{R}^{n}$. Let $\varphi \in \Phi_{w}(\Omega)$ satisfy (AO), (Al) and (aDec). If $v \in W^{1, \varphi(\cdot)}(\Omega)$ and $\operatorname{spt} v \subset \Omega$, then $v \in W_{0}^{1, \varphi(\cdot)}(\Omega)$. 
Proof. Since spt $v \subset \Omega$ and spt $v$ is closed, we can find $\Omega^{\prime} \subset \Omega$ which is bounded and quasiconvex and contains spt $v$. Since values outside $\Omega^{\prime}$ do not affect the claim, we may consider the problem in $\Omega^{\prime}$. To simplify notation, we assume without loss of generality that $\Omega$ is bounded and quasiconvex. Then Lemma 5.1 and Theorem 6.6 of [20] imply that $C^{\infty}(\Omega) \cap W^{1, \varphi(\cdot)}(\Omega)$ is dense in $W^{1, \varphi(\cdot)}(\Omega)$. Let $v \in W^{1, \varphi(\cdot)}(\Omega)$ with spt $v \subset \Omega$. Let $\xi_{i}$ be a sequence in $C^{\infty}(\Omega)$ converging to $v$ in $W^{1, \varphi(\cdot)}(\Omega)$. Let $\eta \in C_{0}^{\infty}(\Omega)$ be a cut-off function with $0 \leqslant \eta \leqslant 1$ and $\eta \equiv 1$ in spt $v$. Then $\eta \xi_{i}$ is a sequence in $C_{0}^{\infty}(\Omega)$. Since $v-\eta \xi_{i}=\eta\left(v-\xi_{i}\right)$, a short calculation gives that $\eta \xi_{i} \rightarrow v$ in $W^{1, \varphi(\cdot)}(\Omega)$ and thus $v \in W_{0}^{1, \varphi(\cdot)}(\Omega)$.

Next we prove a modular maximal inequality which holds only for functions with range $\{0\} \cup[1, \infty)$, and a modular-type Sobolev inequality in the Orlicz space (i.e. without direct $x$-dependence). It can be shown by a scaling argument that such modular inequalities cannot hold in the generalized Orlicz case without extra restrictions. Later on, these will be used to estimate the large and small parts of our quasiminimizers, respectively.

Lemma 3.5. Suppose that $\varphi \in \Phi_{w}(3 Q)$ satisfies (AO), (AI) and (aInc). There exists $\beta>0$ such that

$$
\int_{Q} \varphi\left(x, \beta M f_{1}\right) d x \lesssim \int_{Q} \varphi\left(x, f_{1}\right) d x
$$

for all $f_{1} \in L^{\varphi(\cdot)}(3 Q)$ with norm at most 1 , support in $Q$ and range in $\{0\} \cup[1, \infty)$. The implicit constant depends only on the parameters in assumptions (AO), (Al) and (aInc) and the dimension $n$.

Proof. By Proposition 2.2 of [20] there exists a convex $\psi \in \Phi(3 Q)$ such that $\psi \simeq \varphi$ which satisfies $\sigma$-versions of (A0), (A1) and (aInc). Note that if $x \in Q$ and $B(x, r)$ does not cover $Q$, then $B(x, r) \subset 3 Q$. Since $f_{1}$ has support in $Q$, it follows that it suffices to consider balls in $3 Q$ in the maximal operator. Thus we can conclude as in the proof of Lemma 4.4 of [25] that

$$
\left(\psi\left(x, \frac{\beta}{2} f_{B} f_{1} d x\right)\right)^{\frac{1}{\gamma^{-}}} \leqslant f_{B} \psi\left(x, f_{1}\right)^{\frac{1}{\gamma^{-}}} \leqslant M\left(\psi\left(\cdot, f_{1}\right)^{\frac{1}{\gamma^{-}}}\right)(x)
$$

for $\left\|f_{1}\right\|_{L^{\psi(\cdot)(3 Q)}} \leqslant 1$. Taking supremum over all balls, we find that

$$
\left(\psi\left(x, \frac{\beta}{2} M f_{1}\right)\right)^{\frac{1}{\gamma^{-}}} \leqslant M\left(\psi\left(\cdot, f_{1}\right)^{\frac{1}{\gamma^{-}}}\right)(x) .
$$

Raising both sides to the power $\gamma^{-}$, integrating the over $Q$ and using that $M: L^{\gamma^{-}} \rightarrow L^{\gamma^{-}}$ is bounded, we obtain that

$$
\int_{Q} \psi\left(x, \frac{\beta}{2} M f_{1}\right) d x \leqslant \int_{Q} M\left(\psi\left(\cdot, f_{1}\right)^{\frac{1}{\gamma^{-}}}\right)(x)^{\gamma^{-}} d x \lesssim \int_{Q} \psi\left(x, f_{1}\right) d x .
$$

Since $\varphi \simeq \psi$ with a constant $L$, we obtain that

$$
\int_{Q} \varphi\left(x, \frac{\beta M f_{1}}{2 L^{2}}\right) d x \leqslant \int_{Q} \psi\left(x, \frac{\beta M f_{1}}{2 L}\right) d x \lesssim \int_{Q} \psi\left(x, \frac{f_{1}}{L}\right) d x \leqslant \int_{Q} \varphi\left(x, f_{1}\right) d x .
$$

Note that in the next lemma, $\varphi$ is independent of $x$.

Lemma 3.6. If $Q \subset \mathbb{R}^{n},|Q| \leqslant V$, and $\varphi \in \Phi_{w}$ is doubling, then

$$
\int_{Q} \varphi(v)^{n^{\prime}} d x \lesssim\left(\int_{Q} \varphi(|\nabla v|) d x\right)^{n^{\prime}}
$$

for all $v \in W_{0}^{1, \varphi}(Q)$. The implicit constant depends only on $n$ and $V$. 
Proof. By Lemma 2.3 there exists a convex continuous $\psi \in \Phi_{w}$ such that $\psi \simeq \varphi$. Since $\varphi$ is doubling, $\psi \approx \varphi$ and thus it is enough to prove the claim for convex and continuous $\varphi$.

We first show that there exists $\bar{\varphi} \in C^{1} \cap \Phi_{w}$ such that $\varphi \approx \bar{\varphi}$. Define $\bar{\varphi}(0):=0$ and

$$
\bar{\varphi}(t):=\frac{1}{t} \int_{0}^{t} \varphi(s) d s \text { for } t>0 .
$$

Since $\varphi$ is doubling, convex and increasing, $\varphi(t) \lesssim \frac{1}{2} \varphi\left(\frac{t}{2}\right) \leqslant \bar{\varphi}(t) \leqslant \varphi(t)$. Hence $\bar{\varphi} \approx \varphi$ and so $\bar{\varphi} \in \Phi_{w}$. For $t>0$ we have

$$
\bar{\varphi}^{\prime}(t)=\frac{1}{t} \varphi(t)-\frac{1}{t^{2}} \int_{0}^{t} \varphi(s) d s=\frac{\varphi(t)-\bar{\varphi}(t)}{t} .
$$

Thus $\bar{\varphi}^{\prime}(\cdot)$ is continuous in $(0, \infty)$. To investigate the continuity at 0 , we set $a:=\lim _{t \rightarrow 0^{+}} \frac{\varphi(t)}{t}$ (which exists since $\frac{\varphi(t)}{t}$ is increasing) and $\epsilon(t):=\frac{\varphi(t)}{t}-a$. Then $\epsilon(t) \geqslant 0$ and $\lim _{t \rightarrow 0^{+}} \epsilon(t)=$ 0 . Note that $\bar{\varphi}^{\prime}(0)=\lim _{t \rightarrow 0^{+}} \frac{\bar{\varphi}(t)}{t}$. Thus continuity of the derivative requires that $\frac{\varphi(t)-2 \bar{\varphi}(t)}{t} \rightarrow$ 0 . We calculate

$$
\begin{aligned}
\lim _{t \rightarrow 0^{+}} \frac{\varphi(t)-2 \bar{\varphi}(t)}{t} & =\lim _{t \rightarrow 0^{+}}\left(a+\epsilon(t)-\frac{2}{t^{2}} \int_{0}^{t} a s+\epsilon(s) s d s\right) \\
& =\lim _{t \rightarrow 0^{+}} \epsilon(t)-\lim _{t \rightarrow 0^{+}} \frac{2}{t^{2}} \int_{0}^{t} \epsilon(s) s d s=-\lim _{t \rightarrow 0^{+}} \frac{2}{t^{2}} \int_{0}^{t} \epsilon(s) s d s .
\end{aligned}
$$

Since $\epsilon(t)$ is increasing in $t$, we find that

$$
0 \leqslant \lim _{t \rightarrow 0^{+}} \frac{2}{t^{2}} \int_{0}^{t} \epsilon(s) s d s \leqslant \lim _{t \rightarrow 0^{+}} \frac{2}{t^{2}} \epsilon(t) \int_{0}^{t} s d s=\lim _{t \rightarrow 0^{+}} \epsilon(t)=0 .
$$

Hence we obtain continuity at the origin also, so that $\bar{\varphi}(\cdot) \in C^{1}([0, \infty))$. Next we show that $\bar{\varphi}(s) / s$ is increasing:

$$
\begin{aligned}
\frac{d}{d s} \frac{\bar{\varphi}(s)}{s} & =\frac{\varphi(s)-2 \bar{\varphi}(s)}{s^{2}}=\frac{1}{s^{2}}\left(\varphi(s)-\frac{2}{s} \int_{0}^{s} \varphi(t) d t\right) \\
& \geqslant \frac{1}{s^{2}}\left(\varphi(s)-\frac{2}{s} \frac{\varphi(s)}{s} \int_{0}^{s} t d t\right)=0 .
\end{aligned}
$$

Let $v \in C_{0}^{1}(\Omega)$. It follows from the classical $L^{1}$-Sobolev inequality that

$$
\left(\int_{Q} \bar{\varphi}(v)^{n^{\prime}} d x\right)^{\frac{1}{n^{\prime}}} \lesssim \int_{Q}|\nabla \bar{\varphi}(v)| d x .
$$

By our above expression for the derivative,

$$
\bar{\varphi}^{\prime}(t)=\frac{\varphi(t)-\bar{\varphi}(t)}{t} \leqslant \frac{\varphi(t)}{t} \lesssim \frac{\bar{\varphi}(t)}{t} .
$$

Furthermore,

$$
\bar{\varphi}^{*}\left(\frac{\bar{\varphi}(t)}{t}\right)=\sup _{s>0}\left(\frac{\bar{\varphi}(t)}{t}-\frac{\bar{\varphi}(s)}{s}\right) s=\sup _{s \in(0, t]}\left(\frac{\bar{\varphi}(t)}{t}-\frac{\bar{\varphi}(s)}{s}\right) s \leqslant \sup _{s \in(0, t]} \frac{\bar{\varphi}(t)}{t} s \leqslant \bar{\varphi}(t),
$$

where the second equality holds since $\frac{\bar{\varphi}(t)}{t}$ is increasing. By Young's inequality (2.4) and these estimates,

$$
|\nabla(\bar{\varphi}(v))|=\bar{\varphi}^{\prime}(v)|\nabla v| \lesssim \frac{\bar{\varphi}(v)}{v}|\nabla v| \leqslant \epsilon \bar{\varphi}^{*}\left(\frac{\bar{\varphi}(v)}{v}\right)+c_{\epsilon} \bar{\varphi}(|\nabla v|) \leqslant \epsilon \bar{\varphi}(v)+c_{\epsilon} \bar{\varphi}(|\nabla v|) .
$$


Thus we have by Hölder's inequality

$$
\begin{aligned}
\left(\int_{Q} \bar{\varphi}(v)^{n^{\prime}} d x\right)^{\frac{1}{n^{\prime}}} & \lesssim \epsilon \int_{Q} \bar{\varphi}(v) d x+c_{\epsilon} \int_{Q} \bar{\varphi}(\nabla v) d x \\
& \lesssim \epsilon V^{\frac{1}{n}}\left(\int_{Q} \bar{\varphi}(v)^{n^{\prime}} d x\right)^{\frac{1}{n^{\prime}}}+c_{\epsilon} \int_{Q} \bar{\varphi}(|\nabla v|) d x
\end{aligned}
$$

Choosing $\epsilon$ small enough, we can absorb the first term on the right-hand side into the lefthand side and obtain the claim for $v \in C_{0}^{1}(\Omega)$. Since $W_{0}^{1, \varphi(\cdot)}(\Omega)$ is a closure of $C_{0}^{\infty}(\Omega)$ in $W^{1, \varphi(\cdot)}(\Omega)$, the general claim follows from this by a standard approximation argument.

\section{LOCAL BOUNDEDNESS}

We use the following setup for the rest of this paper.

Notation 4.1. Suppose that $0 \in \Omega \subset \mathbb{R}^{n}$ and $0<R<R_{0} \leqslant \frac{1}{2}$. We write $Q_{R}:=Q(0, R)$ for the cube centered at 0 with side-length $2 R$,

$$
A_{R}:=A(k, R):=Q_{R} \cap\{u>k\} \quad \text { and } \quad u_{+}:=\max \{u, 0\} .
$$

Once we have our results for cubes centered at 0 , we can get the general result by translation. Note that the $\Phi$-function also has to be translated, since our space is not translationinvariant as such.

We need the following lemma which is a generalization from Giusti's book [16, Lemma 6.1, p. 191]. In the original version, $X(t)=t^{p}+t^{q}$.

Lemma 4.2. Let $Z$ be a bounded non-negative function in the interval $[r, R] \subset \mathbb{R}$ and let $X$ be a doubling function in $[0, \infty)$. Assume that there exists $\theta \in[0,1)$ such that

$$
Z(t) \leqslant X\left(\frac{1}{s-t}\right)+\theta Z(s)
$$

for all $r \leqslant t<s \leqslant R$. Then

$$
Z(r) \lesssim X\left(\frac{1}{R-r}\right)
$$

where the implicit constant depends only on the doubling constant and $\theta$.

Proof. Let $L$ be the doubling constant of $X, Q:=\log _{2}(L)$ and $a \in\left(\theta^{1 / Q}, 1\right)$. Define $r_{0}:=r$, $\delta_{i}:=(R-r)(1-a) a^{i}$ and $r_{i}:=r_{i-1}+\delta_{i}$. Iterating the assumption, we find that

$$
Z(r) \leqslant X\left(\frac{1}{\delta_{1}}\right)+\theta Z\left(r_{1}\right) \leqslant \cdots \leqslant \sum_{k=1}^{\infty} \theta^{k-1} X\left(\frac{1}{\delta_{k}}\right)+\lim _{k \rightarrow \infty} \theta^{k} Z\left(r_{k}\right) .
$$

Since $Z$ is bounded, the limit equals zero. Since $X$ is doubling, (2.2) implies that

$$
X\left(\frac{1}{\delta_{k}}\right)=X\left(\frac{1}{(1-a) a^{k}} \frac{1}{R-r}\right) \leqslant L^{2}\left((1-a) a^{k}\right)^{-Q} X\left(\frac{1}{R-r}\right) .
$$

Thus we find that

$$
Z(r) \leqslant \sum_{k=1}^{\infty} \frac{L^{2} \theta^{k-1} X\left(\frac{1}{R-r}\right)}{\left((1-a) a^{k}\right)^{Q}}=\frac{L^{2} X\left(\frac{1}{R-r}\right)}{\theta(1-a)^{Q}} \sum_{k=1}^{\infty}\left(\frac{\theta}{a^{Q}}\right)^{k}=\frac{L^{2} X\left(\frac{1}{R-r}\right)}{(1-a)^{Q}\left(a^{Q}-\theta\right)}
$$

Lemma 4.3 (Caccioppoli inequality). Let $\varphi \in \Phi_{w}(\Omega)$ be doubling. Let u be a local quasiminimizer in $\Omega$. Then for all $k \in \mathbb{R}$ we have

$$
\int_{A(k, r)} \varphi\left(x,\left|\nabla(u-k)_{+}\right|\right) d x \lesssim \int_{A(k, R)} \varphi\left(x, \frac{u-k}{R-r}\right) d x,
$$

where the implicit constant depends only on the doubling constant of $\varphi$ and the quasiminimizing constant of $u$. 
Proof. Let $r \leqslant t<s \leqslant R$ and $k \in \mathbb{R}$. Let $\eta \in C_{0}^{\infty}\left(Q_{s}\right)$ be such that $0 \leqslant \eta \leqslant 1, \eta=1$ in $Q_{t}$, and $|\nabla \eta| \leqslant \frac{2}{s-t}$. Denote $w:=(u-k)_{+}$and $v:=u-\eta w$. Note that $v \leqslant u$, and $v=u$ in $Q_{s} \backslash A_{s}$. Since $u$ is a local quasiminimizer with constant $K$ and $\operatorname{spt}(u-v) \subset Q_{s}$,

$$
\int_{A_{s}} \varphi(x,|\nabla u|) d x \leqslant K \int_{A_{s}} \varphi(x,|\nabla v|) d x .
$$

In $A_{s}, w=u-k$ so that $v=u(1-\eta)+\eta k$, and hence $\nabla v=(1-\eta) \nabla u-(u-k) \nabla \eta$. From this follows that in $A_{s}$ we have

$$
|\nabla v| \leqslant(1-\eta)|\nabla u|+|\nabla \eta|(u-k)_{+} \leqslant 2 \max \left\{(1-\eta)|\nabla u|,|\nabla \eta|(u-k)_{+}\right\} .
$$

By doubling (with constant $L$ ) and $|\nabla \eta| \leqslant \frac{2}{s-t}$, we get that

$$
\begin{aligned}
\varphi(x,|\nabla v|) & \leqslant \varphi(x, 2(1-\eta)|\nabla u|)+\varphi\left(x, 4 \frac{(u-k)_{+}}{s-t}\right) \\
& \leqslant L \varphi(x,(1-\eta)|\nabla u|)+L^{2} \varphi\left(x, \frac{(u-k)_{+}}{s-t}\right) .
\end{aligned}
$$

Combining the above inequalities, we find that

$$
\int_{A_{s}} \varphi(x,|\nabla u|) d x \leqslant L K \int_{A_{s}} \varphi(x,(1-\eta)|\nabla u|) d x+L^{2} K \int_{A_{s}} \varphi\left(x, \frac{(u-k)_{+}}{s-t}\right) d x .
$$

Since $t<s<R$, it follows that $A_{t} \subset A_{s} \subset A_{R}$, and so we obtain

$$
\int_{A_{t}} \varphi(x,|\nabla u|) d x \leqslant L K \int_{A_{s}} \varphi(x,(1-\eta)|\nabla u|) d x+L^{2} K \int_{A_{R}} \varphi\left(x, \frac{(u-k)_{+}}{s-t}\right) d x .
$$

On the right-hand side, we have $\varphi(x,(1-\eta)|\nabla u|)=\varphi(x, 0)=0$ in $Q_{t}$, and so

$$
\int_{A_{s}} \varphi(x,(1-\eta)|\nabla u|) d x=\int_{A_{s} \backslash A_{t}} \varphi(x,(1-\eta)|\nabla u|) d x \leqslant \int_{A_{s} \backslash A_{t}} \varphi(x,|\nabla u|) d x .
$$

Now we can use the hole-filling trick by adding $L K \int_{A_{t}} \varphi(x,|\nabla u|) d x$ to both sides of (4.5), ending with $L K+1$ of the integral on the left-hand side, and $L K$ on the right. After we divide with $L K+1$, we have

$$
\int_{A_{t}} \varphi(x,|\nabla u|) d x \leqslant \frac{L K}{L K+1} \int_{A_{s}} \varphi(x,|\nabla u|) d x+\frac{L^{2} K}{L K+1} \int_{A_{R}} \varphi\left(x, \frac{(u-k)_{+}}{s-t}\right) d x .
$$

The multiplier $\frac{L K}{L K+1}<1$, so the claim follows from Lemma 4.2 with $Z(\tau):=\int_{A_{\tau}} \varphi(x,|\nabla u|) d x$ and $X(\tau):=\frac{L^{2} K}{L K+1} \int_{A_{R}} \varphi\left(x, \tau(u-k)_{+}\right) d x$.

The following is the main technical lemma of the paper. It follows the general scheme of having a larger exponent on the right-hand side, which will allow us to iterate later on. Several of the technical details are quite complicated and novel, however.

Lemma 4.6. Let $\varphi \in \Phi_{w}(\Omega)$ satisfy (AO), (A1), (aInc) and (aDec). Suppose that $u \in$ $W_{\text {loc }}^{1, \varphi(\cdot)}(\Omega)$ satisfies the Caccioppoli inequality (4.4). Let $\frac{R}{2} \leqslant \sigma<\tau \leqslant R$ with $Q_{3 R} \subset \Omega$ and define $\bar{\varphi}(x, t):=\varphi_{Q_{R}}^{-}(t) \chi_{[0,1]}(t)+\varphi(x, t) \chi_{(1, \infty)}(t)$. There exists $R_{0}$ such that

$$
\int_{Q_{\sigma}} \bar{\varphi}\left(x,(u-k)_{+}\right) d x \lesssim \int_{Q_{\tau}} \bar{\varphi}\left(x, \frac{(u-h)_{+}}{\tau-\sigma}\right) d x\left(\int_{Q_{\tau}} \frac{\bar{\varphi}\left(x,(u-h)_{+}\right)}{\bar{\varphi}(x, k-h)} d x\right)^{\alpha},
$$

when $R \in\left(0, R_{0}\right]$ and $k-h \geqslant \tau-\sigma$, where $\alpha:=\frac{\gamma^{-}}{n^{2} \gamma^{+}-n\left(\gamma^{+}-\gamma^{-}\right)}$.

Here $R_{0}$ is such that $R_{0} \leqslant c(n)$ and $\varrho_{L^{\varphi(\cdot)}\left(Q_{3 R_{0}}\right)}(\nabla u) \leqslant 1$, and the implicit constant depends only on the parameters in assumptions (AO), (A1), (aInc) and (aDec), and n. 
Proof. Let $h<k, Q:=Q_{(\sigma+\tau) / 2}$ and $\eta \in C_{0}^{\infty}(Q)$ be a cut-off function such that $0 \leqslant \eta \leqslant 1$, $\eta=1$ in $Q_{\sigma}$, and $|\nabla \eta| \leqslant \frac{4}{\tau-\sigma}$. Denote $v:=(u-k)_{+} \eta, A:=\{v>1\} \cap Q, B:=\{v \leqslant 1\} \cap Q$ and $D:=\{u>k\} \cap Q$. Then

$$
\int_{Q_{\sigma}} \bar{\varphi}\left(x,(u-k)_{+}\right) d x \leqslant \int_{Q} \bar{\varphi}\left(x,(u-k)_{+} \eta\right) d x=\int_{A} \bar{\varphi}(x, v) d x+\int_{B} \bar{\varphi}(x, v) d x .
$$

Denote by $n^{\prime}$ the Hölder conjugate of the dimension $n$. Define $\epsilon:=\left(n^{\prime}-1\right) \frac{\gamma^{-}}{\gamma^{+}}$. If $t>1$, then (aInc), (aDec), and (A0) imply that

$$
\bar{\varphi}\left(x, t^{n^{\prime}}\right) \gtrsim t^{\left(n^{\prime}-1\right) \gamma^{-}} \bar{\varphi}(x, t) \gtrsim\left(\frac{\bar{\varphi}(x, t)}{\bar{\varphi}(x, 1)}\right)^{\left(n^{\prime}-1\right) \gamma^{-} / \gamma^{+}} \bar{\varphi}(x, t) \approx \bar{\varphi}(x, t)^{1+\epsilon} .
$$

With Hölder's inequality, we then estimate

$$
\int_{A} \bar{\varphi}(x, v) d x \lesssim \int_{A} \bar{\varphi}\left(x, v^{n^{\prime}}\right)^{\frac{1}{1+\epsilon}} d x \leqslant|A|^{\frac{\epsilon}{1+\epsilon}}\left(\int_{A} \bar{\varphi}\left(x, v^{n^{\prime}}\right) d x\right)^{\frac{1}{1+\epsilon}} .
$$

Denote by $I$ the Riesz potential operator and set $f_{1}:=|\nabla v| \chi_{\{|\nabla v|>1\}}$. Since $v \in W_{0}^{1,1}(Q)$ we obtain by the Riesz potential estimate, see for example [15, Lemma 7.14, p. 161], that

$$
v \leqslant c_{1} I(\nabla v) \leqslant c_{1} I\left(\nabla v \chi_{\{|\nabla v| \leqslant 1\}}\right)+c_{1} I\left(f_{1}\right) .
$$

Now $I\left(\nabla v \chi_{\{|\nabla v| \leqslant 1\}}\right) \leqslant I\left(\chi_{Q}\right) \leqslant c R_{0} \leqslant \frac{1}{2 c_{1}}$, provided $R_{0}$ is small enough. Since $v>1$ in $A$, we obtain that $v \leqslant \frac{1}{2} v+c_{1} I\left(f_{1}\right)$ and hence $v \lesssim I\left(f_{1}\right)$ in $A$. Suppose that $R_{0}$ is so small that $\|\nabla u\|_{L^{\varphi(\cdot)}(3 Q)} \leqslant 1$. Since $\left\|f_{1}\right\|_{L^{1}\left(\mathbb{R}^{n}\right)}=\left\|f_{1}\right\|_{L^{1}(3 Q)} \leqslant\|\nabla u\|_{L^{\varphi(\cdot)}(3 Q)} \leqslant 1$, it follows that $\left(I f_{1}\right)^{n^{\prime}} \leqslant c M f_{1}[23,(3)$, p. 506] and that Lemma 3.5 is applicable. Since $\varphi$ is doubling, the constant $\beta$ in the lemma can be transferred outside $\varphi$. Combining the previous estimates and the lemma for the last step, we find that

$$
\int_{A} \bar{\varphi}\left(x, v^{n^{\prime}}\right) d x \lesssim \int_{A} \bar{\varphi}\left(x,\left(I f_{1}\right)^{n^{\prime}}\right) d x \lesssim \int_{A} \bar{\varphi}\left(x, M f_{1}\right) d x \lesssim \int_{A} \bar{\varphi}(x, \nabla v) d x .
$$

Extend $\left.\bar{\varphi}\right|_{[0,1]}$ to $(1, \infty)$ as $\varphi_{Q_{R}}^{+}(1) t^{\gamma^{-}}$and call the result $\widetilde{\varphi}$. By (aInc) and (A0), $\widetilde{\varphi}(t) \lesssim$ $\bar{\varphi}(x, t)$ for every $x$. We next estimate the measure of $A=\{v>1\} \cap Q$. By (A0), $\chi_{A} \lesssim$ $\widetilde{\varphi}(v)^{n^{\prime}}$ and by Lemmas 3.4 and 3.6 (for the second inequality):

$$
|A| \lesssim \int_{Q} \widetilde{\varphi}(v)^{n^{\prime}} d x \lesssim\left(\int_{Q} \widetilde{\varphi}(|\nabla v|) d x\right)^{n^{\prime}} \lesssim\left(\int_{Q} \bar{\varphi}(x,|\nabla v|) d x\right)^{n^{\prime}},
$$

On the other hand, $|A| \leqslant|D|$, so that $|A|^{\frac{\epsilon}{1+\epsilon}} \leqslant|D|^{\frac{\epsilon}{n(1+\epsilon)}}|A|^{\frac{\epsilon}{n^{\prime}(1+\epsilon)}}$. Collecting the estimates for the integral over $A$ and using $\bar{\varphi} \leqslant \varphi$, we obtain from (4.7) and (4.8) that

$$
\int_{A} \bar{\varphi}(x, v) d x \lesssim|D|^{\frac{\epsilon}{n(1+\epsilon)}}\left(\int_{Q} \varphi(x,|\nabla v|) d x\right)^{\frac{n^{\prime} \epsilon}{n^{\prime}(1+\epsilon)}+\frac{1}{1+\epsilon}}
$$

Denote the exponent of $|D|$ by $\alpha:=\frac{\epsilon}{n(1+\epsilon)}$ and note that the exponent of the integral equals 1 .

Let us turn to the estimate of the integral in the set $B$. In this case the function $\bar{\varphi}$ is independent of $x$ and equals $\widetilde{\varphi}$. By Hölder's inequality,

$$
\int_{B} \bar{\varphi}(x, v) d x \leqslant \int_{D} \widetilde{\varphi}(v) d x \lesssim|D|^{\frac{1}{n}}\left(\int_{Q} \widetilde{\varphi}(v)^{n^{\prime}} d x\right)^{\frac{1}{n^{\prime}}} .
$$


Since $|D| \leqslant|Q| \leqslant 1$ and $\alpha=\frac{\epsilon}{n(1+\epsilon)} \leqslant \frac{1}{n}$, we see that $|D|^{\frac{1}{n}} \leqslant|D|^{\alpha}$. By Lemma 3.4 and 3.6 and $\widetilde{\varphi} \lesssim \bar{\varphi} \leqslant \varphi$, we continue the estimate and find that

$$
\int_{B} \bar{\varphi}(x, v) d x \lesssim|D|^{\alpha} \int_{Q} \widetilde{\varphi}(|\nabla v|) d x \leqslant|D|^{\alpha} \int_{Q} \varphi(x,|\nabla v|) d x
$$

We have now estimated the integral over both set $A$ and set $B$ by the same upper bound, which we next consider further.

By the product rule, $|\nabla v| \leqslant\left|\nabla(u-k)_{+}\right|+(u-k)_{+}|\nabla \eta|$. Since $|\nabla \eta| \leqslant \frac{4}{\tau-\sigma}$, we obtain by this and the Caccioppoli inequality (4.4) that

$$
\begin{aligned}
\int_{Q} \varphi(x,|\nabla v|) d x & \lesssim \int_{Q} \varphi\left(x,\left|\nabla(u-k)_{+}\right|\right)+\varphi\left(x, \frac{(u-k)_{+}}{\tau-\sigma}\right) d x \\
& \lesssim \int_{Q_{\tau}} \varphi\left(x, \frac{(u-k)_{+}}{\tau-\sigma}\right) d x
\end{aligned}
$$

where the doubling of $\varphi$ was also used. In the set $D=\{u>k\} \cap Q$, we observe that $(u-h)_{+} \geqslant k-h \geqslant \tau-\sigma$. Hence

$$
\varphi\left(x, \frac{(u-k)_{+}}{\tau-\sigma}\right) \leqslant \varphi\left(x, \frac{(u-h)_{+}}{\tau-\sigma}\right) \chi_{D} \leqslant \bar{\varphi}\left(x, \frac{(u-h)_{+}}{\tau-\sigma}\right) .
$$

From $(u-h)_{+} \geqslant k-h$ in $D$ we also derive, since $\bar{\varphi}$ is increasing, that

$$
|D|=\int_{Q_{\tau}} \chi_{D} d x \leqslant \int_{Q_{\tau}} \frac{\bar{\varphi}\left(x,(u-h)_{+}\right)}{\bar{\varphi}(x, k-h)} d x .
$$

Collecting all our estimates, we conclude that

$$
\int_{Q} \bar{\varphi}(x, v) d x \lesssim \int_{Q_{\tau}} \bar{\varphi}\left(x, \frac{(u-h)_{+}}{\tau-\sigma}\right) d x\left(\int_{Q_{\tau}} \frac{\bar{\varphi}\left(x,(u-h)_{+}\right)}{\bar{\varphi}(x, k-h)} d x\right)^{\alpha} .
$$

Remark 4.9. Note from the proof that we could also have replaced

$$
\int_{Q_{\tau}} \frac{\bar{\varphi}\left(x,(u-h)_{+}\right)}{\bar{\varphi}(x, k-h)} d x \quad \text { by } \quad \int_{Q_{\tau}} \bar{\varphi}\left(x, \frac{(u-h)_{+}}{k-h}\right) d x
$$

since, by $(\mathrm{A} 0), \bar{\varphi}\left(x, \frac{(u-h)_{+}}{k-h}\right) \geqslant 1$ in $D$. However, in our next derivations the unusual form chosen works better than the alternative, more standard form.

Now that our estimate is done, we use standard iteration based on the following lemma.

Lemma 4.10 (Lemma 7.1, [16]). Let $\alpha>0$ and $\left(\Phi_{i}\right)$ be a sequence of real numbers such that

$$
\Phi_{i+1} \leqslant D L^{i} \Phi_{i}^{1+\alpha}
$$

with $D>0$ and $L>1$. If $\Phi_{0} \leqslant D^{-1 / \alpha} L^{-1 / \alpha^{2}}$, then $\Phi_{i} \rightarrow 0$ as $i \rightarrow \infty$.

We can now present a preliminary version of the ess sup-estimate. In this estimate there is an extra " +1 ", a bad dependence on the size $R$ of the cube, and an inhomogeneity on the right-hand side. We will remove the latter two deficiencies later. Nevertheless, even the preliminary estimate allows us to conclude that every local quasiminimizer is locally bounded. Note that we do not need the assumption (A1- $n$ ) for this result. 
Theorem 4.11. Let $\varphi \in \Phi_{w}(\Omega)$ satisfy (AO), (A1), (aInc) and (aDec). Suppose that $u \in$ $W_{\mathrm{loc}}^{1, \varphi(\cdot)}(\Omega)$ satisfies the Caccioppoli inequality (4.4) and that $R_{0} \in(0,1)$ is as in Lemma 4.6. Then

$$
\underset{Q_{R / 2}}{\operatorname{ess} \sup } u \leqslant k_{0}+1+c R^{-\frac{\gamma^{+}}{\alpha \gamma^{-}}}\left(\int_{Q_{R}} \varphi\left(x,\left(u-k_{0}\right)_{+}\right) d x\right)^{\frac{1}{\gamma^{-}}}
$$

for every $k_{0} \in \mathbb{R}$ when $R \in\left(0, R_{0}\right]$. Here the constant $c$ depends only on the parameters in assumptions (AO), (AI), (aInc) and (aDec) and the dimension $n$.

Proof. Let $d \geqslant 1$ be a number to be determined later. We choose levels $k_{i}:=k_{0}+d\left(1-2^{-i}\right)$ and radii $\sigma_{i}:=\frac{R}{2}\left(1+2^{-i}\right), i \in \mathbb{N}$. Note that $k_{i}$ increases from $k_{0}$ to $k_{0}+d$, and $\sigma_{i}$ decreases from $R$ to $R / 2$. Let $\bar{\varphi}$ be as in Lemma 4.6 and define

$$
\Phi_{i}:=\int_{Q_{\sigma_{i}}} \bar{\varphi}\left(x,\left(u-k_{i}\right)_{+}\right) d x .
$$

Observe that $\varphi\left(x, d 2^{-i-1}\right) \gtrsim d^{\gamma^{-}} 2^{-(i+1) \gamma^{+}}$by (aDec), (aInc) and (A0). By Lemma 4.6 with $k:=k_{i+1}, h:=k_{i}, \sigma:=\sigma_{i+1}$ and $\tau:=\sigma_{i}$,

$$
\begin{aligned}
\Phi_{i+1} & \lesssim \int_{Q_{\sigma_{i}}} \bar{\varphi}\left(x, \frac{\left(u-k_{i}\right)_{+}}{\sigma_{i}-\sigma_{i+1}}\right) d x\left(\int_{Q_{\sigma_{i}}} \frac{\bar{\varphi}\left(x,\left(u-k_{i}\right)_{+}\right)}{\bar{\varphi}\left(x, k_{i+1}-k_{i}\right)} d x\right)^{\alpha} \\
& =\int_{Q_{\sigma_{i}}} \bar{\varphi}\left(x, \frac{\left(u-k_{i}\right)_{+}}{R 2^{-i-2}}\right) d x\left(\int_{Q_{\sigma_{i}}} \frac{\bar{\varphi}\left(x,\left(u-k_{i}\right)_{+}\right)}{\bar{\varphi}\left(x, d 2^{-i-1}\right)} d x\right)^{\alpha} \\
& \lesssim R^{-\gamma^{+}} 2^{i(1+\alpha) \gamma^{+}} d^{-\alpha \gamma^{-}} \Phi_{i}^{1+\alpha},
\end{aligned}
$$

where we used $(\mathrm{aDec})$ and the previous estimate for the second inequality.

Thus the inequality from Lemma 4.10 holds with $D:=c R^{-\gamma^{+}} d^{-\alpha \gamma^{-}}$and $L:=2^{(1+\alpha) \gamma^{+}}$. The required condition $\Phi_{0} \leqslant D^{-1 / \alpha} L^{-1 / \alpha^{2}}$ is therefore

$$
\Phi_{0} \leqslant c R^{\frac{\gamma^{+}}{\alpha}} d^{\gamma^{-}} 2^{-\frac{1+\alpha}{\alpha^{2}} \gamma^{+}}
$$

Collecting the restrictions on $d$, we see that we may choose $d=\max \left\{1, c R^{-\frac{\gamma^{+}}{\alpha \gamma^{-}}} \Phi_{0}^{\frac{1}{\gamma^{-}}}\right\}$. With this choice, Lemma 4.10 implies that

$$
\int_{Q_{\sigma_{\infty}}} \bar{\varphi}\left(x,\left(u-k_{\infty}\right)_{+}\right) d x=\lim _{i \rightarrow \infty} \Phi_{i}=0 .
$$

Thus $u \leqslant k_{\infty}=k_{0}+d$ almost everywhere in $Q_{\sigma_{\infty}}=Q_{R / 2}$. Since $\bar{\varphi} \leqslant \varphi, \Phi_{0}$ can be estimated by the integral in the statement of the theorem, so the claim follows.

\section{IMPROVED UPPER BOUND FOR BOUNDED SOLUTIONS}

We can easily and with minimal assumptions derive the following version of Lemma 4.6. It is worse than the previous estimate in that the left-hand side contains $\varphi_{Q_{R}}^{-}$, but this will not matter since we will use it for bounded functions. On the positive side, we do not need to assume any smallness of the norm of the gradient.

Lemma 5.1. Let $\varphi \in \Phi_{w}(\Omega)$ satisfy $(A 0)$ and (aDec) and define $\widetilde{\varphi}(x, t):=t^{\gamma^{+}} \varphi^{-}(1) \chi_{[0,1)}(t)+$ $\varphi(x, t) \chi_{[1, \infty)}(t)$. Suppose that $u \in W_{\mathrm{loc}}^{1, \varphi(\cdot)}(\Omega)$ satisfies the Caccioppoli inequality (4.4). Let $\frac{R}{2} \leqslant \sigma<\tau \leqslant R$ with $Q_{R} \subset \Omega$. Then

$$
\int_{Q_{\sigma}} \widetilde{\varphi}_{Q_{R}}^{-}\left((u-k)_{+}\right) d x \lesssim\left(\int_{Q_{\tau}} \frac{\widetilde{\varphi}\left(x,(u-h)_{+}\right)}{\widetilde{\varphi}(x, k-h)} d x\right)^{\frac{1}{n}} \int_{Q_{\tau}} \widetilde{\varphi}\left(x, \frac{(u-h)_{+}}{\tau-\sigma}\right) d x
$$


when $k-h \geqslant \tau-\sigma$. The implicit constant depends only on the parameters in assumptions (AO) and (aDec) and on $n$.

Proof. Let $h<k, Q:=Q_{(\sigma+\tau) / 2}$ and $\eta \in C_{0}^{\infty}(Q)$ be a cut-off function such that $0 \leqslant \eta \leqslant 1$, $\eta=1$ in $Q_{\sigma}$, and $|\nabla \eta| \leqslant \frac{4}{\tau-\sigma}$. Denote $v:=(u-k)_{+} \eta$ and $D:=\{u>k\} \cap Q$. By Hölder's inequality,

$$
\int_{Q_{\sigma}} \widetilde{\varphi}_{Q_{R}}^{-}\left((u-k)_{+}\right) d x \leqslant \int_{D} \widetilde{\varphi}_{Q_{R}}^{-}(v) d x \lesssim|D|^{1-\frac{1}{n^{\prime}}}\left(\int_{D} \widetilde{\varphi}_{Q_{R}}^{-}(v)^{n^{\prime}} d x\right)^{\frac{1}{n^{\prime}}} .
$$

By definition and (aDec), $\widetilde{\varphi}_{Q_{R}}^{-}(t) \leqslant \varphi(x, t)$. Sobolev's inequality (Lemma 3.6) and this estimate yield

$$
\int_{Q_{\sigma}} \widetilde{\varphi}_{Q_{R}}^{-}\left((u-k)_{+}\right) d x \lesssim|D|^{\frac{1}{n}} \int_{D} \widetilde{\varphi}_{Q_{R}}^{-}(|\nabla v|) d x \lesssim|D|^{\frac{1}{n}} \int_{D} \varphi(x,|\nabla v|) d x .
$$

Exactly as in Lemma 4.6, we derive

$$
\int_{Q} \varphi(x,|\nabla v|) d x \lesssim \int_{Q_{\tau}} \varphi\left(x, \frac{(u-k)_{+}}{\tau-\sigma}\right) d x \lesssim \int_{Q_{\tau}} \widetilde{\varphi}\left(x, \frac{(u-h)_{+}}{\tau-\sigma}\right) d x
$$

and

$$
|D|=\int_{Q_{\tau}} \chi_{D} d x \leqslant \int_{Q_{\tau}} \frac{\widetilde{\varphi}\left(x,(u-h)_{+}\right)}{\widetilde{\varphi}(x, k-h)} d x
$$

from which the claim follows.

Even though the $\varphi(\cdot)$-Laplace equation is not very scalable, it is possible to scale the solution provided we also modify $\varphi$. Let us define, for $s>0$,

$$
u_{s}(x):=\frac{u(s x)}{s} \text { and } \varphi_{s}(x, f(x)):=\varphi(s x, f(x)) .
$$

Note that $\varphi_{s}$ has the same doubling constant as $\varphi$. The constants of (A1) and (A1-n) are not invariant under this scaling, so they have to be considered separately where needed. Recall that $0 \in \Omega$ and we study cubes centered at 0 .

Lemma 5.2. Let $\varphi \in \Phi_{w}(\Omega)$ and let $u \in W_{\mathrm{loc}}^{1, \varphi(\cdot)}(\Omega)$ be a local $K$-quasiminimizer of the $\varphi$-energy. Let $s \in(0,1]$ and suppose that $Q_{s R} \Subset \Omega$. Then $u_{s}$ is a K-quasiminimizer of the $\varphi_{s}$-energy in $Q_{R}$.

Proof. Note first that $\nabla u_{s}(x)=\nabla u(s x)$. Let $v_{s} \in W^{1, \varphi(\cdot)}\left(Q_{R}\right)$ with spt $v_{s} \subset Q_{R}$ and observe that $\{v \neq 0\}=s\left\{v_{s} \neq 0\right\} \subset Q_{s R}$. Two changes of variables and the quasiminimizing property of $u$ in $Q_{s R}$ yield

$$
\begin{gathered}
\int_{\left\{v_{s} \neq 0\right\}} \varphi_{s}\left(x,\left|\nabla u_{s}(x)\right|\right) d x=\int_{\left\{v_{s} \neq 0\right\}} \varphi_{s}(x,|\nabla u(s x)|) d x=s^{-n} \int_{\{v \neq 0\}} \varphi(z,|\nabla u(z)|) d z \\
\leqslant K s^{-n} \int_{\{v \neq 0\}} \varphi(z,|\nabla(u+v)(z)|) d z=K \int_{\left\{v_{s} \neq 0\right\}} \varphi_{s}\left(x,\left|\nabla\left(u_{s}+v_{s}\right)(x)\right|\right) d x
\end{gathered}
$$

Since $u_{s}$ is a quasiminimizer of the $\varphi_{s}$-energy, we could directly apply Lemma 4.6 to it. However, this would incur the restriction $\varrho_{L^{\varphi_{s}(\cdot)}\left(Q_{R}\right)}\left(\nabla u_{s}\right) \leqslant 1$. Undoing the scaling, this is equivalent to $\varrho_{L^{\varphi}(\cdot)}\left(Q_{s R}\right)(\nabla u) \leqslant s^{n}$, which is a problem when $s \rightarrow 0$. Therefore, we use Lemma 5.1 instead. Note that the constant in the next proposition depends on $\|u\|_{\infty}$, not $\left\|u_{s}\right\|_{\infty}$ 
Proposition 5.3. Let $\varphi \in \Phi_{w}(\Omega)$ satisfy (AO), (Al-n) and (aDec). Suppose that $u \in$ $W_{\mathrm{loc}}^{1, \varphi(\cdot)}(\Omega)$ is a bounded local quasiminimizer. Then

$$
\underset{Q_{R / 2}}{\operatorname{ess} \sup } u_{s}-k_{0} \lesssim\left(\int_{Q_{R}}\left(u_{s}-k_{0}\right)_{+}^{\gamma^{+}} d x\right)^{\frac{1}{\gamma^{+}}}+R
$$

when $0<R<R_{0}, s \in(0,1], Q_{s R} \Subset \Omega$ and $k_{0}>-2\left\|u_{s}\right\|_{L^{\infty}\left(Q_{R}\right)}$. The constant $R_{0}$ depends only on $n$ and $\|u\|_{L^{\infty}\left(Q_{s R}\right)}$. The implicit constant is independent of $s$ and depends only on the parameters in assumptions (AO), (Al-n) and (aDec) as well as on $n, R$ and $\|u\|_{L^{\infty}\left(Q_{s R}\right)}$.

Proof. Denote $\widetilde{\varphi}(x, t):=t^{\gamma^{+}} \varphi^{-}(1) \chi_{[0,1)}(t)+\varphi(x, t) \chi_{[1, \infty)}(t)$ and suppose that $t_{0} \in\left[0, \frac{3\|u\|_{\infty}}{s}\right]$. Undoing the scaling, we see that

$$
\left(\widetilde{\varphi}_{s}\right)_{Q_{R}}^{+}\left(\beta t_{0}\right) \leqslant\left(\widetilde{\varphi}_{s}\right)_{Q_{R}}^{-}\left(t_{0}\right) \quad \Leftrightarrow \quad \widetilde{\varphi}_{Q_{s R}}^{+}\left(\beta t_{0}\right) \leqslant \widetilde{\varphi}_{Q_{s R}}^{-}\left(t_{0}\right)
$$

If $t_{0}<\frac{1}{\beta}$, then the inequality is trivially true by the definition of $\widetilde{\varphi},(\mathrm{A} 0)$ and $(\mathrm{aDec})$. When $t_{0} \geqslant \frac{1}{\beta}$, by $(\mathrm{A} 1-n)$ and Lemma 2.10 , the latter inequality holds when $t_{0} \leqslant \frac{1}{2 \sqrt{n} s R}$. Since $t_{0} \leqslant \frac{3\|u\|_{\infty}}{s}$, a sufficient condition is $\frac{3\|u\|_{\infty}}{s} \leqslant \frac{1}{2 \sqrt{n} s R}$ which holds provided $R_{0}<\frac{1}{6 \sqrt{n}\|u\|_{\infty}}$.

Let $d_{0}:=\max \left\{\left(\int_{Q_{R}}\left(u_{s}-k_{0}\right)_{+}^{\gamma^{+}} d x\right)^{\frac{1}{\gamma^{+}}}, R\right\}$ and $d:=K d_{0}$ where the constant $K \geqslant 1$ will be specified later. Note that $d_{0} \in\left[0, \frac{3\|u\|_{\infty}}{s}\right]$. Let us denote $\psi:=\left(\widetilde{\varphi}_{s}\right)_{Q_{R}}^{+}$. By the almost increasing property of $\frac{\varphi(x, t)}{t},(5.4)$ and doubling,

$$
\widetilde{\varphi}_{s}(x, d) \gtrsim K \widetilde{\varphi}_{s}\left(x, d_{0}\right) \geqslant K \psi\left(\beta d_{0}\right) \gtrsim K \psi\left(d_{0}\right) .
$$

For $i \in \mathbb{N}$, let $k_{i}:=k_{0}+d\left(1-2^{-i}\right)$ and $\sigma_{i}:=\frac{R}{2}\left(1+2^{-i}\right)$. Note that $k_{i+1}-k_{i} \geqslant \sigma_{i}-\sigma_{i+1}$. Define

$$
\Phi_{i}:=\int_{Q_{\sigma_{i}}} \widetilde{\varphi}_{s}\left(x,\left(u_{s}-k_{i}\right)_{+}\right) d x
$$

By (5.4), (A0) and (aDec),

$$
\Phi_{i+1} \lesssim \int_{Q_{\sigma_{i+1}}}\left(\widetilde{\varphi}_{s}\right)_{Q_{R}}^{-}\left(\left(u_{s}-k_{i+1}\right)_{+}\right) d x .
$$

By Lemma 5.2, the function $u_{s}$ is a local quasiminimizer and thus by Lemma 4.3 it satisfies the Caccioppoli inequality (4.4). Hence by Lemma 5.1

$$
\begin{aligned}
\Phi_{i+1} & \lesssim\left(\int_{Q_{\sigma_{i}}} \frac{\widetilde{\varphi}_{s}\left(x,\left(u_{s}-k_{i}\right)_{+}\right)}{\widetilde{\varphi}_{s}\left(x, k_{i+1}-k_{i}\right)} d x\right)^{\frac{1}{n}} \int_{Q_{\sigma_{i}}} \widetilde{\varphi}_{s}\left(x, \frac{\left(u_{s}-k_{i}\right)_{+}}{\sigma_{i}-\sigma_{i+1}}\right) d x \\
& =\left(\int_{Q_{\sigma_{i}}} \frac{\left.\widetilde{\varphi}_{s}\left(x, u_{s}(x)-k_{i}\right)_{+}\right)}{\widetilde{\varphi}_{s}\left(x, d 2^{-i-1}\right)} d x\right)^{\frac{1}{n}} \int_{Q_{\sigma_{i}}} \widetilde{\varphi}_{s}\left(x, \frac{\left(u_{s}(x)-k_{i}\right)_{+}}{R 2^{-i-2}}\right) d x
\end{aligned}
$$

By (aDec) and almost increasing inherent in $\Phi_{w}, \widetilde{\varphi}_{s}\left(x, K d_{0} 2^{-i-1}\right) \gtrsim K 2^{-(i+1) \gamma^{+}} \widetilde{\varphi}_{s}\left(x, d_{0}\right)$ and

$$
\widetilde{\varphi}_{s}\left(x, \frac{\left(u_{s}(x)-k_{i}\right)_{+}}{R 2^{-i-2}}\right) \lesssim\left(R 2^{-i-2}\right)^{-\gamma^{+}} \widetilde{\varphi}_{s}\left(x,\left(u_{s}(x)-k_{i}\right)_{+}\right)
$$


Taking also into account (5.5), the previous estimate can be continued by

$$
\begin{aligned}
\Phi_{i+1} & \lesssim\left(\int_{Q_{\sigma_{i}}} \frac{\left.\widetilde{\varphi}_{s}\left(x, u_{s}(x)-k_{i}\right)_{+}\right)}{2^{-(i+1) \gamma^{+}} K \psi\left(d_{0}\right)} d x\right)^{\frac{1}{n}} \int_{Q_{\sigma_{i}}}\left(R 2^{-i-2}\right)^{-\gamma^{+}} \widetilde{\varphi}_{s}\left(x,\left(u_{s}(x)-k_{i}\right)_{+}\right) d x \\
& =\left[2^{-(i+1) \gamma^{+}} K \psi\left(d_{0}\right)\right]^{-\frac{1}{n}}\left(R 2^{-i-2}\right)^{-\gamma^{+}} \Phi_{i}^{1+\frac{1}{n}} \\
& \approx 2^{i \gamma^{+}\left(1+\frac{1}{n}\right)} K^{-\frac{1}{n}} \psi\left(d_{0}\right)^{-\frac{1}{n}} R^{-\gamma^{+}} \Phi_{i}^{1+\frac{1}{n}} .
\end{aligned}
$$

Thus the inequality from Lemma 4.10 holds with $\alpha:=\frac{1}{n}, D:=c R^{-\gamma^{+}} K^{-\alpha} \psi\left(d_{0}\right)^{-\alpha}$ and $L:=2^{(1+\alpha) \gamma^{+}}$. The condition of the lemma now requires that

$$
\Phi_{0} \leqslant c 2^{-\frac{1+\alpha}{\alpha^{2}} \gamma^{+}} R^{\frac{\gamma^{+}}{\alpha}} K \psi\left(d_{0}\right)
$$

Then the lemma implies that $u \leqslant k_{\infty}=k_{0}+K d_{0}$ almost everywhere in $Q_{\sigma_{\infty}}=Q_{R / 2}$, which gives the claim of the proposition.

We will show that $\Phi_{0} / \psi\left(d_{0}\right)$ is bounded independent of $s$. Then we can choose $K$ to ensure that inequality (5.6) holds. Since $t^{-\gamma^{+}} \widetilde{\varphi}_{s}(x, t)$ is almost decreasing, the same holds for $\psi$. Then, by Lemma 2.8, $t^{-1} \psi^{-1}(t)^{\gamma^{+}}$is almost increasing and so $\left(\psi^{-1}\right)^{\gamma^{+}}$is equivalent to a convex function $\xi$ [20, Lemma 2.2]. Since $\widetilde{\varphi}_{s} \leqslant \psi$, it follows from Jensen's inequality that

$$
\begin{aligned}
\psi^{-1}\left(\Phi_{0}\right) & \leqslant \psi^{-1}\left(\int_{Q_{R}} \psi\left(\left(u_{s}-k_{0}\right)_{+}\right) d x\right) \approx\left[\xi\left(\int_{Q_{R}} \psi\left(\left(u_{s}-k_{0}\right)_{+}\right) d x\right)\right]^{\frac{1}{\gamma^{+}}} \\
& \leqslant\left(\int_{Q_{R}} \xi\left(\psi\left(\left(u_{s}-k_{0}\right)_{+}\right)\right) d x\right)^{\frac{1}{\gamma^{+}}} \approx\left(\int_{Q_{R}}\left(u_{s}-k_{0}\right)_{+}^{\gamma^{+}} d x\right)^{\frac{1}{\gamma^{+}}} \leqslant d_{0} .
\end{aligned}
$$

Since $\psi$ is doubling, this yields that $\Phi_{0} \leqslant \psi\left(c d_{0}\right) \lesssim \psi\left(d_{0}\right)$, so $\Phi_{0} / \psi\left(d_{0}\right)$ is bounded, as required.

Theorem 5.7. Let $\varphi \in \Phi_{w}(\Omega)$ satisfy $(A 0),(A 1-n)$ and (aDec). Suppose that $u \in W_{\mathrm{loc}}^{1, \varphi(\cdot)}(\Omega)$ is a bounded local quasiminimizer. Then

$$
\underset{Q_{r / 2}}{\operatorname{ess} \sup } u-k \lesssim\left(f_{Q_{r}}(u-k)_{+}^{\gamma^{+}} d x\right)^{\frac{1}{\gamma^{+}}}+r
$$

when $r \in\left(0, R_{0}\right]$ and $k \in \mathbb{R}$. The implicit constant depends only on the parameters in assumptions (AO), (Al-n) and (aDec), $n, R_{0}$ and $\|u\|_{L^{\infty}\left(Q_{r}\right)}$.

Proof. Suppose first that $k_{0} \geqslant-2\left\|u_{s}\right\|_{\infty}$ i.e. $k:=s k_{0} \geqslant-2\|u\|_{\infty}$. In Proposition 5.3 we fix $R=R_{0}$ and multiply the result by $s \in(0,1]$ :

$$
\underset{Q_{s R_{0} / 2}}{\operatorname{ess} \sup } u-k=\underset{Q_{R_{0} / 2}}{\operatorname{ess} \sup } s u_{s}-k \lesssim\left(\int_{Q_{R_{0}}}\left(s u_{s}-k\right)_{+}^{\gamma^{+}} d x\right)^{\frac{1}{\gamma^{+}}}+R_{0} s
$$

where the implicit constant is independent of $s$. Next we change variables $\left(x:=s^{-1} z\right)$ :

$$
\int_{Q_{R_{0}}}\left(s u_{s}(x)-k\right)_{+}^{\gamma^{+}} d x=s^{-n} \int_{Q_{s R_{0}}}(u(z)-k)_{+}^{\gamma^{+}} d z=c f_{Q_{s R_{0}}}(u(z)-k)_{+}^{\gamma_{+}^{+}} d z,
$$

where the constant depends on $R_{0}$ but not $s$. Hence we obtain that

$$
\underset{Q_{s R_{0} / 2}}{\operatorname{ess~sup}} u-k \lesssim\left(f_{Q_{s R_{0}}}(u-k)_{+}^{\gamma^{+}} d z\right)^{\frac{1}{\gamma^{+}}}+R_{0} s \text {. }
$$


Denoting $r:=s R_{0} \in\left(0, R_{0}\right]$, we obtain that

$$
\underset{Q_{r / 2}}{\operatorname{ess} \sup } u-k \lesssim\left(f_{Q_{r}}(u-k)_{+}^{\gamma^{+}} d z\right)^{\frac{1}{\gamma^{+}}}+r .
$$

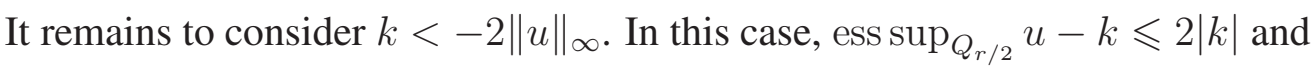

$$
\left(f_{Q_{r}}(u-k)_{+}^{\gamma^{+}} d x\right)^{\frac{1}{\gamma^{+}}} \geqslant \frac{1}{2}\left(f_{Q_{r}}|k|^{\gamma^{+}} d x\right)^{\frac{1}{\gamma^{+}}}=\frac{|k|}{2}
$$

so the claim again holds.

Instead of the cube $Q_{R / 2}$, it is possible to have any cube smaller than $Q_{R}$ on the right-hand side. For that we use cubes not centered at the origin.

Corollary 5.8. Let $\varphi \in \Phi_{w}(\Omega), u \in W_{\mathrm{loc}}^{1, \varphi(\cdot)}(\Omega)$ and $R_{0}>0$ be as in Theorem 5.7. For every $\tau \in(0,1)$

$$
\underset{Q_{\tau R}}{\operatorname{ess} \sup } u-k \lesssim\left(\frac{1}{(1-\tau)^{n}} f_{Q_{R}}(u-k)_{+}^{\gamma^{+}} d x\right)^{\frac{1}{\gamma^{+}}}+R
$$

when $R \in\left(0, R_{0}\right]$ and $k \in \mathbb{R}$. The implicit constant is independent of $R$ and $\tau$.

Proof. Let us choose $x_{1} \in Q_{\tau R}$ such that $\underset{Q_{\tau R}}{\operatorname{ess} \sup } u=\underset{Q\left(x_{1},(1-\tau) R / 2\right)}{\operatorname{ess} \sup } u$. By Theorem 5.7,

$$
\operatorname{esssup}_{Q\left(x_{1},(1-\tau) R / 2\right)} u-k \lesssim\left(f_{Q\left(x_{1},(1-\tau) R\right)}(u-k)_{+}^{\gamma^{+}} d x\right)^{\frac{1}{\gamma^{+}}}+(1-\tau) R .
$$

Since $Q\left(x_{1},(1-\tau) R\right) \subset Q_{R}$, we obtain that

$$
\operatorname{esssup}_{Q\left(x_{1},(1-\tau) R / 2\right)} u-k \lesssim\left(\frac{1}{(1-\tau)^{n}} f_{Q_{R}}(u-k)_{+}^{\gamma^{+}} d x\right)^{\frac{1}{\gamma^{+}}}+R .
$$

Corollary 5.9. Let $\varphi \in \Phi_{w}(\Omega), u \in W_{\mathrm{loc}}^{1, \varphi(\cdot)}(\Omega)$ and $R_{0}>0$ be as in Theorem 5.7. Then

$$
\underset{Q_{R / 2}}{\operatorname{ess} \sup } u-k \lesssim\left(f_{Q_{R}}(u-k)_{+}^{q} d x\right)^{\frac{1}{q}}+R,
$$

for every $R \in\left(0, R_{0}\right], k \in \mathbb{R}$ and $q \in(0, \infty)$. The implicit constant is independent of $R$ and depends on $q$ and on the parameters listed in Theorem 5.7.

Proof. If $q \geqslant \gamma^{+}$, then the claim follows from Theorem 5.7 by Hölder's inequality. So we may assume that $0<q<\gamma^{+}$.

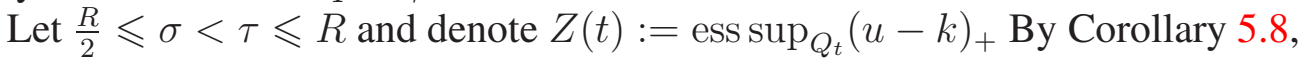

$$
Z(\sigma) \lesssim\left(\frac{1}{(1-\sigma / \tau)^{n}} f_{Q_{\tau}}(u-k)_{+}^{\gamma^{+}} d x\right)^{\frac{1}{\gamma^{+}}}+R=\left(\frac{c}{(\tau-\sigma)^{n}} \int_{Q_{\tau}}(u-k)_{+}^{\gamma^{+}} d x\right)^{\frac{1}{\gamma^{+}}}+R .
$$

Thus we obtain by Young's inequality with exponents $\frac{\gamma^{+}}{q}$ and $\frac{\gamma^{+}}{\gamma^{+}-q}=: \frac{1}{\theta}$ that

$$
\begin{aligned}
Z(\sigma) & \lesssim\left(\frac{1}{(\tau-\sigma)^{n}} \int_{Q_{\tau}}(u-k)_{+}^{q} d x\right)^{\frac{1}{\gamma^{+}}} Z(\tau)^{\theta}+R \\
& \leqslant \underbrace{\frac{c q}{\gamma^{+}}\left(\frac{1}{(\tau-\sigma)^{n}} \int_{Q_{R}}(u-k)_{+}^{q} d x\right)^{\frac{1}{q}}+R}_{=: X\left(\frac{1}{\tau-\sigma}\right)}+\theta Z(\tau) .
\end{aligned}
$$


Then $Z$ is bounded in $[R / 2, R], X$ is doubling and $Z(\sigma) \leqslant X\left(\frac{1}{\tau-\sigma}\right)+\theta Z(\tau)$ for all $\frac{R}{2} \leqslant$ $\sigma<\tau \leqslant R$. Hence Lemma 4.2 yields $Z\left(\frac{R}{2}\right) \lesssim X\left(\frac{2}{R}\right)$, which is the claim.

\section{THE WEAK HARNACK INEQUALITY}

In this section, we denote $D_{\theta}:=\{u<\theta\} \cap Q_{R}$.

Lemma 6.1. If $u \geqslant 0,-u$ satisfies (5.10), with $q=1$ and constant $c_{1}$, and

$$
\left|D_{\theta}\right| \leqslant \frac{1}{2 c_{1}}\left|Q_{R}\right|
$$

for some $\theta>0$, then

$$
\underset{Q_{R / 2}}{\operatorname{essinf}} u+c_{1} R \geqslant \frac{\theta}{2}
$$

Proof. Equation (5.10) applied to $-u$ with $k=-\theta$ and $q=1$ gives

$$
\begin{aligned}
\underset{Q_{R / 2}}{\operatorname{ess} \sup }(-u) & \leqslant-\theta+c_{1} f_{Q_{R}}(\theta-u)_{+} d x+c_{1} R \\
& \leqslant-\theta+c_{1} \theta \frac{\left|D_{\theta}\right|}{\left|Q_{R}\right|}+c_{1} R \leqslant-\frac{\theta}{2}+c_{1} R .
\end{aligned}
$$

The following is the main lemma of this section.

Lemma 6.2. Let $\varphi \in \Phi_{w}(\Omega), u \in W_{\mathrm{loc}}^{1, \varphi(\cdot)}(\Omega)$ and $R_{0}>0$ be as in Theorem 1.4. If $u \geqslant 0$, then for every $\kappa \in(0,1)$ there exists $\mu>0$ such that

$$
\left|D_{\theta}\right| \leqslant \kappa\left|Q_{R}\right| \Rightarrow \underset{Q_{R / 2}}{\operatorname{essinf}} u+c R \geqslant \mu \theta
$$

for all $R \in\left(0, R_{0}\right]$ and all $\theta>0$.

Proof. By Lemma 2.3, we may assume without loss of generality that $\varphi$ is a bijection. We assume first that $\theta>R$. Abbreviate $Q:=Q_{R}$ and set, for $R<h<k<\theta$,

$$
v= \begin{cases}0, & \text { if } u \geqslant k \\ k-u, & \text { if } h<u<k \\ k-h, & \text { if } u \leqslant h\end{cases}
$$

Then $v \in W_{\text {loc }}^{1, \varphi(\cdot)}(\Omega)$ and $|\nabla v|=|\nabla u| \chi_{\{h<u<k\}}$ a.e. in $\Omega$.

Clearly $v=0$ in $Q \backslash D_{\theta}$, and since $\left|D_{\theta}\right| \leqslant \kappa|Q|$, we have

$$
\left|Q \backslash D_{\theta}\right| \geqslant(1-\kappa)|Q| \text {. }
$$

Under these circumstances, [16, Theorem 3.16] tells us that

$$
\left(\int_{Q} v^{n^{\prime}} d x\right)^{\frac{1}{n^{\prime}}} \leqslant C(n, \kappa)|\Delta| f_{\Delta}|\nabla v| d x
$$

for $v \in W^{1,1}(Q)$ and $\Delta:=D_{k} \backslash D_{h}$. By Hölder's inequality,

$$
(k-h)\left|D_{h}\right|^{\frac{1}{n^{\prime}}}=\left|D_{h}\right|^{-\frac{1}{n}} \int_{D_{h}} v d x \leqslant\left(\int_{Q} v^{n^{\prime}} d x\right)^{\frac{1}{n^{\prime}}} \lesssim|\Delta| f_{\Delta}|\nabla v| d x .
$$

Denote $V(x):=\varphi(x,|\nabla v(x)|)$. By [24, Lemma 4.3],

$$
\frac{1}{2} f_{\Delta}|\nabla v| d x \leqslant\left(\varphi_{Q}^{-}\right)^{-1}\left(f_{\Delta} \varphi_{Q}^{-}(|\nabla v|) d x\right) \leqslant\left(\varphi_{Q}^{-}\right)^{-1}\left(f_{\Delta} V d x\right) .
$$


Since $\varphi$ satisfies (aInc), so does $\varphi_{Q}^{-}$. Thus $t^{-\frac{1}{\gamma^{-}}}\left(\varphi_{Q}^{-}\right)^{-1}(x, t)$ is almost decreasing by Lemma 2.8 . This and $\frac{|Q|}{|\Delta|} \geqslant 1$ imply that

$$
\left(\varphi_{Q}^{-}\right)^{-1}\left(f_{\Delta} V d x\right) \lesssim\left(\frac{|Q|}{|\Delta|}\right)^{\frac{1}{\gamma^{-}}}\left(\varphi_{Q}^{-}\right)^{-1}\left(\frac{1}{|Q|} \int_{\Delta} V d x\right)
$$

Suppose first that $f_{Q} V d x \geqslant 1$. Then the inequality $\varrho_{\varphi(\cdot)}(\nabla u) \leqslant c(n)$ implies that the conditions in Lemma 2.11 (based on (A1)) are fulfilled, and so we conclude that

$$
\left(\varphi_{Q}^{-}\right)^{-1}\left(\frac{1}{|Q|} \int_{\Delta} V d x\right) \leqslant\left(\varphi_{2 Q}^{-}\right)^{-1}\left(f_{Q} V d x\right) \leqslant \frac{1}{\beta}\left(\varphi_{2 Q}^{+}\right)^{-1}\left(f_{Q} V d x\right) .
$$

Since $u$ is a local quasiminimizer so is $-u$ and thus by the Caccioppoli estimate (4.4) and (aInc) we have

$$
f_{Q} V d x=f_{Q} \varphi(x,|\nabla v|) d x \leqslant c f_{2 Q} \varphi\left(x, \frac{v}{R}\right) d x \leqslant \varphi_{2 Q}^{+}\left(c_{1} \frac{k}{R}\right) .
$$

If $f_{Q} V d x \leqslant 1$, then the inequality $\left(\varphi_{2 Q}^{-}\right)^{-1}\left(f_{Q} V d x\right) \leqslant \varphi_{2 Q}^{+}\left(c_{1} \frac{k}{R}\right)$ also holds, by (A0) and $\frac{k}{R} \geqslant 1$. Combining the previous five inequality-lines, we find that

$$
(k-h)\left|D_{h}\right|^{\frac{1}{n^{\prime}}} \lesssim|\Delta|\left(\frac{|Q|}{|\Delta|}\right)^{\frac{1}{\gamma^{-}}}\left(\varphi_{2 Q}^{+}\right)^{-1}\left(\varphi_{2 Q}^{+}\left(c_{1} \frac{k}{R}\right)\right) \leqslant c_{1}|\Delta|^{1-\frac{1}{\gamma^{-}}} R^{\frac{n}{\gamma^{-}}} \frac{k}{R} .
$$

Divide the previous inequality by $k$, raise it to the power $\tau:=\frac{\gamma^{-}}{\gamma^{-}-1}$ and substitute $k:=k_{i}$ and $h:=k_{i+1}, i \in \mathbb{N}$ :

$$
\left(\frac{k_{i}-k_{i+1}}{k_{i}}\right)^{\tau}\left|D_{k_{i+1}}\right|^{\frac{\tau}{n^{\prime}}} \leqslant\left[\left|D_{k_{i}}\right|-\left|D_{k_{i+1}}\right|\right] R^{\frac{n-\gamma^{-}}{\gamma^{-}} \tau}
$$

Assume first that $\theta>2^{i_{0}} R$, where $i_{0} \in \mathbb{N}$ will be fixed later. Set $k_{i}:=\theta 2^{-i}$ and $d_{i}:=\left|D_{k_{i}}\right|$ for $i=0, \ldots, i_{0}$ and note that $\frac{k_{i}-k_{i+1}}{k_{i}}=\frac{1}{2}$. Since $d_{i} \geqslant d_{i_{0}}=\left|D_{\theta 2^{-i_{0}}}\right|$ for $i \leqslant i_{0}$, this implies that

$$
\left|D_{\theta 2^{-i} 0}\right|^{\frac{\tau}{n^{\prime}}} \lesssim\left[d_{i}-d_{i+1}\right] R^{\frac{n-\gamma^{-}}{\gamma^{-}} \tau}
$$

Adding these inequalities for $i$ from 0 to $i_{0}$, we get

$$
\left(i_{0}+1\right)\left|D_{\theta 2^{-i}}\right|^{\frac{\tau}{n^{\prime}}} \lesssim R^{\frac{n-\gamma^{-}}{\gamma^{-}} \tau}\left(d_{0}-d_{i_{0}}\right) \lesssim R^{n+\frac{n-\gamma^{-}}{\gamma^{-}} \tau} .
$$

Now $n+\frac{n-\gamma^{-}}{\gamma^{-}} \tau=\gamma^{-} \frac{n-1}{\gamma^{-}-1}=\tau(n-1)$. Hence we obtain

$$
\left|D_{\theta 2^{-i_{0}}}\right| \lesssim\left(i_{0}+1\right)^{-\frac{n^{\prime}}{\tau}} R^{n}=c\left(i_{0}+1\right)^{-\frac{n^{\prime}}{\tau}}|Q|
$$

We choose $i_{0}$ such that $C\left(i_{0}+1\right)^{-\frac{n^{\prime}}{\tau}} \leqslant \frac{1}{2 c_{1}}$, where $c_{1}$ is the constant from Lemma 6.1. Then by Corollary 5.9 and Lemma 6.1, $\operatorname{ess~} \inf _{Q_{R / 2}} u+c R \geqslant \theta 2^{-i_{0}-1}$, so the claim holds with the choice $\mu:=2^{-i_{0}-1}$.

If, on the other hand, $\theta \in\left(0,2^{i_{0}} R\right]$, then

$$
\text { ess inf } u+c R \geqslant c R \geqslant \theta c 2^{-i_{0}}
$$

so the claim holds with $\mu:=c 2^{-i_{0}}$.

The statement of the next lemma is exactly what is proved in [22, Theorem 5.7], although the assumptions are stated differently. For completeness, the proof is included below. 
Lemma 6.3. Assume that $u \geqslant 0$ and $R_{0}$ are such that for every $\kappa>0$, there exist $\mu>0$ with

$$
\left|D_{\theta}\right| \leqslant \kappa\left|Q_{R}\right| \Rightarrow \underset{Q_{R / 2}}{\operatorname{essinf}} u+R \geqslant \mu \theta
$$

for all $R \in\left(0, R_{0}\right)$. Then there exists $q>0$ such that

$$
\left(f_{Q_{R}} u^{q} d x\right)^{\frac{1}{q}} \lesssim \operatorname{essinf}_{Q_{R / 2}} u+R
$$

for every $R \in\left(0, R_{0}\right)$.

Since $\mu$ in Lemma 6.2 depends only on the parameters of Theorem 1.4, we obtain the weak Harnack inequality with the same dependence via the previous lemma.

Corollary 6.5 (Weak Harnack inequality). Let $\varphi \in \Phi_{w}(\Omega)$ satisfy (AO), (A1), (A1-n), (aInc) and (aDec). Suppose that $u \in W_{\mathrm{loc}}^{1, \varphi(\cdot)}(\Omega)$ is a non-negative local quasiminimizer and $R_{0}$ is as in Theorem 5.7. Then

$$
\left(f_{Q_{R}} u^{q} d x\right)^{\frac{1}{q}} \lesssim \underset{Q_{R / 2}}{\operatorname{essinf}} u+R
$$

when $R \in\left(0, R_{0}\right]$ and $k \in \mathbb{R}$. The implicit constant depends only on the parameters in assumptions (AO), (Al), (Al-n), (aInc), (aDec), the dimension $n, R_{0}$ and $\|u\|_{L^{\infty}\left(Q_{R}\right)}$.

For the proof of Lemma 6.3 we need the following covering theorem that is due to Krylov and Safonov [26]. For the proof, see, e.g., the monograph by Giusti [16].

Lemma 6.6. Let $E \subset Q_{R} \subset \mathbb{R}^{n}$ be a measurable set, and let $0<\delta<1$. Moreover, let

$$
E_{\delta}:=\bigcup_{x \in Q_{R}, 0<\varrho<R}\left\{Q(x, 3 \varrho) \cap Q_{R}:|Q(x, 3 \varrho) \cap E| \geqslant \delta\left|Q_{\varrho}\right|\right\} .
$$

Then either $|E| \geqslant \delta\left|Q_{R}\right|$, in which case $E_{\delta}=Q_{R}$, or

$$
\left|E_{\delta}\right| \geqslant \frac{1}{\delta}|E|
$$

In the next proof we denote $D(\theta, z, R):=\{u+R \leqslant \theta\} \cap Q(z, R)$.

Proof of Lemma 6.3. By Cavalieri’s principle,

$$
\int_{Q_{R}}(u+R)^{h} d x=h \int_{0}^{\infty} t^{h-1}\left|A_{t}^{0}\right| d t
$$

where $A_{t}^{0}:=\left\{x \in Q_{R}: u(x)+R>t\right\}$ for $t>0$. In order to estimate the measure of $A_{t}^{0}$, we fix $0<\delta<1$ and $\kappa:=1-\delta / 3^{n}$ and define sets $A_{t}^{i}$ by

$$
A\left(t \mu^{i}, x, R\right):=\left\{y \in Q(x, R): u(y)+R>t \mu^{i}\right\},
$$

where $\mu$ is the constant in (6.4) corresponding the constant $\kappa$. We abbreviate $A_{t}^{i}:=A\left(t \mu^{i}, 0, R\right)$. Suppose that for some $\varrho<R$ and $z \in Q_{R}$, we have

$$
Q(z, 3 \varrho) \cap Q_{R} \subset\left(A_{t}^{i}\right)_{\delta},
$$

where $(\cdot)_{\delta}$ is defined in Lemma 6.6. It follows that

$$
\begin{aligned}
\frac{\delta}{3^{n}}\left|Q_{3 \varrho}\right| & =\delta\left|Q_{\varrho}\right| \leqslant\left|Q(z, 3 \varrho) \cap A\left(t \mu^{i}, 0, R\right)\right| \\
& =\left|Q_{R} \cap A\left(t \mu^{i}, z, 3 \varrho\right)\right| \leqslant\left|A\left(t \mu^{i}, z, 3 \varrho\right)\right| .
\end{aligned}
$$


This estimate implies that

$$
\left|D\left(t \mu^{i}, z, 3 \varrho\right)\right| \leqslant\left(1-\frac{\delta}{3^{n}}\right)\left|Q_{3 \varrho}\right|=\kappa\left|Q_{3 \varrho}\right| .
$$

Hence, (6.4) gives us

$$
\underset{Q(z, 3 \varrho)}{\operatorname{ess} \inf } u+R \geqslant t \mu^{i+1} .
$$

Since assumption (6.8) leads to (6.9), it follows that

$$
\left(A_{t}^{i}\right)_{\delta} \subset A_{t}^{i+1} .
$$

This together with Krylov-Safonov covering lemma (Lemma 6.6) imply that either $A_{t}^{i+1}=$ $Q_{R}$ or $\left|A_{t}^{i+1}\right| \geqslant\left|\left(A_{t}^{i}\right)_{\delta}\right| \geqslant \delta^{-1}\left|A_{t}^{i}\right|$.

If $A_{t}^{i+1}=Q_{R}$, then $\operatorname{essinf}_{Q_{R / 2}} u+R \geqslant t \mu^{i+1}$. Otherwise, $\left|A_{t}^{i+1}\right| \geqslant\left|\left(A_{t}^{i}\right)_{\delta}\right| \geqslant \delta^{-1}\left|A_{t}^{i}\right|$ and so it follows that

$$
\left|A_{t}^{j}\right| \geqslant \delta^{-1}\left|A_{t}^{j-1}\right| \geqslant \delta^{-2}\left|A_{t}^{j-2}\right| \geqslant \ldots \geqslant \delta^{-j}\left|A_{t}^{0}\right| .
$$

If $A_{t}^{0}$ has positive measure we choose $j$ to be the smallest integer satisfying

$$
j \geqslant \frac{1}{\log \delta} \log \frac{\left|A_{t}^{0}\right|}{\left|Q_{R}\right|} .
$$

Then

$$
\delta^{j}\left|Q_{R}\right| \leqslant\left|A_{t}^{0}\right| \leqslant \delta^{j-1}\left|Q_{R}\right|
$$

and thus $\left|A_{t}^{j}\right| \geqslant\left|Q_{R}\right|$ i.e. $A_{t}^{j}=Q_{R}$. As before this leads to ess $\inf _{Q_{R / 2}} u+R \geqslant t \mu^{j}$.

We obtain

$$
\underset{Q_{R / 2}}{\operatorname{essinf}} u+R \geqslant t \mu^{j+1}=C t\left(\frac{\left|A_{t}^{0}\right|}{\left|Q_{R}\right|}\right)^{\log \mu / \log \delta}
$$

or, equivalently, by setting $\xi=\operatorname{ess}_{\inf _{Q_{R / 2}}} u+R$ and $a=\frac{\log \delta}{\log \mu}>0$, we get

$$
\left|A_{t}^{0}\right| \leqslant C\left|Q_{R}\right| \xi^{a} t^{-a} \text {. }
$$

We choose $0<h<a$ and obtain by (6.7) that

$$
\begin{aligned}
\int_{Q_{R}}(u+R)^{h} d x & =h \int_{0}^{\xi} t^{h-1}\left|Q_{R}\right| d t+h \int_{\xi}^{\infty} t^{h-1}\left|A_{t}^{0}\right| d t \\
& \leqslant \xi^{h}\left|Q_{R}\right|+C\left|Q_{R}\right| \xi^{a} \int_{\xi}^{\infty} t^{h-a-1} d t=C\left|Q_{R}\right| \xi^{h}
\end{aligned}
$$

which is

$$
\left(\frac{1}{\left|Q_{R}\right|} \int_{Q_{R}}(u+R)^{h} d x\right)^{1 / h} \lesssim \underset{Q_{R / 2}}{\operatorname{essinf}} u+R .
$$

With the estimate $u \leqslant u+R$ we can complete the proof.

\section{REFERENCES}

[1] M.K. Alaouia, T. Nabilab and M. Altanjia: On some new non-linear diffusion models for the image filtering, Applicable Anal. 93 (2014), no. 2, 269-280.

[2] M. Avci and A. Pankov: Multivalued elliptic operators with nonstandard growth, Adv. Nonlinear Anal., to appear. DOI: 10.1515/anona-2016-0043

[3] P. Baroni, M. Colombo and G. Mingione: Harnack inequalities for double phase functionals, Nonlinear Anal. 121 (2015), 206-222.

[4] P. Baroni, M. Colombo and G. Mingione: Non-autonomous functionals, borderline cases and related function classes, St Petersburg Math. J. 27 (2016), 347-379. 
[5] A. Björn and J. Björn: Nonlinear potential theory on metric spaces, EMS Tracts in Mathematics, 17, European Mathematical Society (EMS), Zürich, 2011.

[6] Y. Chen, S. Levine and M. Rao: Variable exponent, linear growth functionals in image restoration, SIAM J. Appl. Math. 66 (2006), no. 4, 1383-1406.

[7] M. Colombo and G. Mingione: Regularity for double phase variational problems, Arch. Ration. Mech. Anal. 215 (2015), no. 2, 443-496.

[8] M. Colombo and G. Mingione: Bounded minimisers of double phase variational integrals, Arch. Ration. Mech. Anal. 218 (2015), no. 1, 219-273.

[9] M. Colombo and G. Mingione: Calderón-Zygmund estimates and non-uniformly elliptic operators, J. Funct. Anal. 270 (2016), 1416-1478.

[10] D. Cruz-Uribe and A. Fiorenza: Variable Lebesgue spaces, Foundations and harmonic analysis, Birkhäuser/Springer, Heidelberg, 2013.

[11] D. Cruz-Uribe and P. Hästö: Extrapolation and interpolation in generalized Orlicz spaces, Preprint (2016).

[12] L. Diening, P. Harjulehto, P. Hästö and M. Růžička: Lebesgue and Sobolev spaces with variable exponents, Lecture Notes in Mathematics, 2017. Springer, Heidelberg, 2011.

[13] X.-L. Fan: An imbedding theorem for Musielak-Sobolev spaces, Nonlinear Anal. 75 (2012), no. 4, 19591971.

[14] F. Giannetti and A. Passarelli di Napoli: Regularity results for a new class of functionals with non-standard growth conditions, J. Differential Equations 254 (2013) 1280-1305.

[15] D. Gilbarg and N. S. Trudinger: Elliptic partial differential equations of second order, Grundlehren der Mathematischen Wissenschaften, Vol. 224. Springer-Verlag, Berlin-New York, 1977.

[16] E. Giusti: Direct Methods in the Calculus of Variations, World Scientific, Singapore, 2003.

[17] P. Gwiazda, P. Wittbold, A. Wróblewska and A. Zimmermann: Renormalized solutions of nonlinear elliptic problems in generalized Orlicz spaces, J. Differential Equations 253 (2012), no. 2, 635-666; J. Differential Equations 253 (2012), no. 9, 2734-2738.

[18] P. Gwiazda, P. Wittbold, A. Wróblewska and A. Zimmermann: Renormalized solutions to nonlinear parabolic problems in generalized Musielak-Orlicz spaces, Nonlinear Anal. 129 (2015), 1-36.

[19] P. Harjulehto and P. Hästö: Riesz potential in generalized Orlicz Spaces, Forum Math., to appear. DOI: 10.1515/forum-2015-0239

[20] P. Harjulehto, P. Hästö and R. Klén: Generalized Orlicz spaces and related PDE, Nonlinear Anal. 143 (2016), 155-173.

[21] P. Harjulehto, P. Hästö, V. Latvala and O. Toivanen: Critical variable exponent functionals in image restoration, Appl. Math. Letters 26 (2013), 56-60.

[22] P. Harjulehto, T. Kuusi, T. Lukkari, N. Marola, M. Parviainen: Harnack's inequality for quasiminimizers with non-standard growth conditions, J. Math. Anal. Appl. 344 (2008), no. 1, 504-520.

[23] L.I. Hedberg: On certain convolution inequalities, Proc. Amer. Math. Soc. 36 (1972), 505-510.

[24] P. Hästö: The maximal operator on generalized Orlicz spaces, J. Funct. Anal 269 (2015), no. 12, 40384048.

[25] P. Hästö: Corrigendum to "The maximal operator on generalized Orlicz spaces" [J. Funct. Anal. 269 (2015) 4038-4048], J. Funct. Anal. 271 (2016), no. 1, 240-243.

[26] N. V. Krylov and M. V. Safonov: A property of the solutions of parabolic equations with measurable coefficients, (Russian) Izv. Akad. Nauk SSSR Ser. Mat. 44 (1980), 161-175.

[27] F.-Y. Maeda, Y. Mizuta, T. Ohno and T. Shimomura: Boundedness of maximal operators and Sobolev's inequality on Musielak-Orlicz-Morrey spaces, Bull. Sci. Math. 137 (2013), 76-96.

[28] F.-Y. Maeda, Y. Mizuta, T. Ohno and T. Shimomura: Approximate identities and Young type inequalities in Musielak-Orlicz spaces, Czechoslovak Math. J. 63(138) (2013), no. 4, 933-948.

[29] F.-Y. Maeda, Y. Mizuta, T. Ohno and T. Shimomura: Sobolev and Trudinger type inequalities on grand Musielak-Orlicz-Morrey spaces, Ann. Acad. Sci. Fenn. Math. 40 (2015), no. 1, 403-426.

[30] F.-Y. Maeda, T. Ohno and T. Shimomura: Boundedness of the maximal operator on Musielak-OrliczMorrey spaces, Tohoku Math. J., to appear.

[31] P. Marcellini: Regularity of minimizers of integrals of the calculus of variations with nonstandard growth conditions, Arch. Rational Mech. Anal. 105 (1989), no. 3, 267-284.

[32] P. Marcellini: Regularity and existance of solutions of elliptic equations with $p, q$-growth conditions, J. Differential Equations 50 (1991), no. 1, 1-30.

[33] G. Mingione: Regularity of minima: An invitation to the dark side of the calculus of variations, Appl. Math. 51 (2006), no. 4, 355-426. 
[34] J. Musielak: Orlicz spaces and modular spaces, Lecture Notes in Mathematics, 1034. Springer, Berlin, 1983.

[35] T. Ohno and T. Shimomura: Trudinger's inequality for Riesz potentials of functions in Musielak-Orlicz spaces, Bull. Sci. Math. 138 (2014), no. 2, 225-235.

[36] T. Ohno and T. Shimomura: Musielak-Orlicz-Sobolev spaces on metric measure spaces, Czechoslovak Math. J. 65(140) (2015), no. 2, 435-474.

[37] J. Ok: Gradient estimates for elliptic equations with $L^{p(\cdot)} \log L$ growth, Calc. Var. Partial Differential Equations 55 (2016), no. 2, 1-30.

[38] J. Ok: Regularity results for a class of obstacle problems with nonstandard growth, J. Math. Anal. Appl. 444 (2016), no. 2, 957-979.

[39] L. Pick and M. Růžička: An example of a space $L^{p(x)}$ on which the Hardy-Littlewood maximal operator is not bounded, Expo. Math. 19 (2001), no. 4, 369-371.

[40] M. Růžička: Electrorheological fluids: modeling and mathematical theory, Lecture Notes in Mathematics, 1748. Springer-Verlag, Berlin, 2000.

[41] A. Świerczewska-Gwiazda: Nonlinear parabolic problems in Musielak-Orlicz spaces, Nonlinear Anal. 98 (2014), 48-65.

[42] D. Yang, W. Yuan and C. Zhuo: Musielak-Orlicz Besov-type and Triebel-Lizorkin-type spaces, Rev. Mat. Complut. 27 (2014), no. 1, 93-157.

[43] V.V. Zhikov: Averaging of functionals of the calculus of variations and elasticity theory, Izv. Akad. Nauk SSSR Ser. Mat. 50 (1986), 675-710.

[44] V.V. Zhikov: On Lavrentiev's Phenomenon, Russian J. Math. Phys. 3 (1995), 249-269.

P. HARJULEHTO

Department of Mathematics and Statistics, FI-20014 University of Turku, Finland

petteri.harjulehtodutu.fi

\section{P. HÄSTÖ}

Department of Mathematics and Statistics, FI-20014 University of Turku, Finland Department of Mathematics, FI-90014 University of Oulu, Finland

peter.hastodoulu.fi

\section{O. TOIVANEN}

Institute of Mathematics, Polish Academy of Sciences, 00-956 Warsaw, Poland

otoivanen@impan.pl 